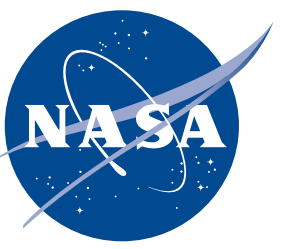

\title{
Performance Theory of Diagonal Conducting Wall Magnetohydrodynamic Accelerators
}

R.J. Litchford

Marshall Space Flight Center, Marshall Space Flight Center, Alabama 


\section{The NASA STI Program Office...in Profile}

Since its founding, NASA has been dedicated to the advancement of aeronautics and space science. The NASA Scientific and Technical Information (STI) Program Office plays a key part in helping NASA maintain this important role.

The NASA STI Program Office is operated by Langley Research Center, the lead center for NASA's scientific and technical information. The NASA STI Program Office provides access to the NASA STI Database, the largest collection of aeronautical and space science STI in the world. The Program Office is also NASA's institutional mechanism for disseminating the results of its research and development activities. These results are published by NASA in the NASA STI Report Series, which includes the following report types:

- TECHNICAL PUBLICATION. Reports of completed research or a major significant phase of research that present the results of NASA programs and include extensive data or theoretical analysis. Includes compilations of significant scientific and technical data and information deemed to be of continuing reference value. NASA's counterpart of peer-reviewed formal professional papers but has less stringent limitations on manuscript length and extent of graphic presentations.

- TECHNICAL MEMORANDUM. Scientific and technical findings that are preliminary or of specialized interest, e.g., quick release reports, working papers, and bibliographies that contain minimal annotation. Does not contain extensive analysis.

- CONTRACTOR REPORT. Scientific and technical findings by NASA-sponsored contractors and grantees.
- CONFERENCE PUBLICATION. Collected papers from scientific and technical conferences, symposia, seminars, or other meetings sponsored or cosponsored by NASA.

- SPECIAL PUBLICATION. Scientific, technical, or historical information from NASA programs, projects, and mission, often concerned with subjects having substantial public interest.

- TECHNICAL TRANSLATION. English-language translations of foreign scientific and technical material pertinent to NASA's mission.

Specialized services that complement the STI Program Office's diverse offerings include creating custom thesauri, building customized databases, organizing and publishing research results...even providing videos.

For more information about the NASA STI Program Office, see the following:

- Access the NASA STI Program Home Page at http://www.sti.nasa.gov

- E-mail your question via the Internet to help@sti.nasa.gov

- Fax your question to the NASA Access Help Desk at (301) 621-0134

- Telephone the NASA Access Help Desk at (301) 621-0390

- Write to: NASA Access Help Desk NASA Center for AeroSpace Information 7121 Standard Drive Hanover, MD 21076-1320 


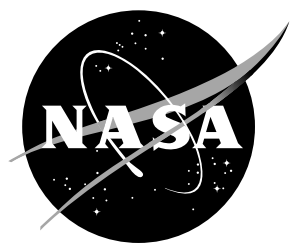

\section{Performance Theory of Diagonal Conducting Wall Magnetohydrodynamic Accelerators}

R.J. Litchford

Marshall Space Flight Center, Marshall Space Flight Center, Alabama

National Aeronautics and

Space Administration

Marshall Space Flight Center • MSFC, Alabama 35812 


\section{Acknowledgments}

The author expresses his thanks to John T. Lineberry, President, LyTec, LLC; Prof. Y.C.L. Susan Wu (retired), University of Tennessee Space Institute; and Prof. Valentin A. Bityurin, Head of MHD Programs, Institute of High Temperatures (IVTAN) of the Russian Academy of Sciences for valuable discussions and for clarifying basic conceptual ideas underlying MHD performance theory.

Available from:

NASA Center for AeroSpace Information

7121 Standard Drive

Hanover, MD 21076-1320

(301) 621-0390
National Technical Information Service 5285 Port Royal Road Springfield, VA 22161 (703) 487-4650 


\section{TABLE OF CONTENTS}

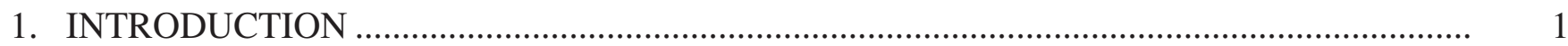

2. CROSSED-FIELD ACCELERATOR CONFIGURATIONS ……………………..................... 3

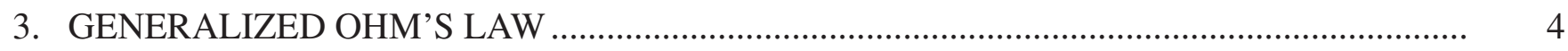

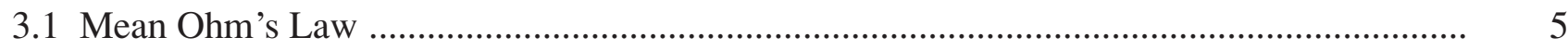

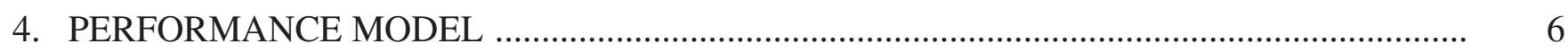

4.1 Diagonal Loading Constraint .............................................................................................. 6

4.2 Electrical Parameter Relationships ........................................................................................ 6

4.3 Mean Power Parameters .................................................................................................... 7

4.3.1 Power Density ...................................................................................................... 7

4.3.2 Push Power ...................................................................................................... 7

4.3.3 Dissipated Power ............................................................................................................ 7

4.3.4 Electrical Efficiency ............................................................................................ 8

4.3.5 Power Relationships ……………............................................................................ 8

4.4 Generalized Vector Diagram ............................................................................................... 8

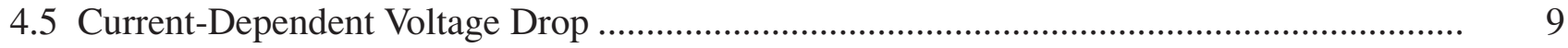

4.5.1 Generator Mode ………………....................................................................... 10

4.5.2 Accelerator Mode ..................................................................................... 10

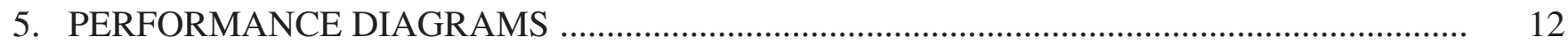

5.1 Dimensionless Performance Parameters ................................................................................ 12

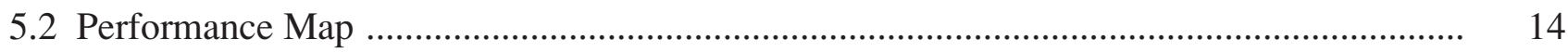

6. ELECTRODE BOUNDARY LAYER EFFECTS …................................................................ 18

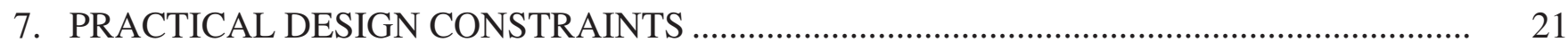

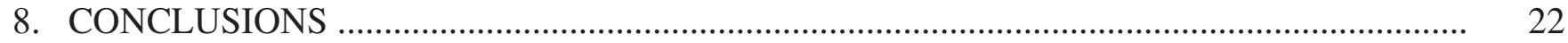

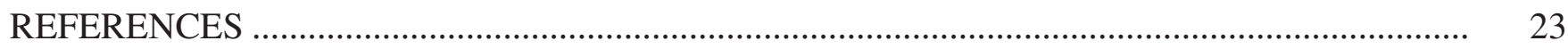




\section{LIST OF FIGURES}

1. Composite graphic of alternative cross-field MHD accelerator configurations:

(a) Linear Hall accelerator, (b) segmented Faraday accelerator, (c) series connected diagonal accelerator, and (d) DCW accelerator.

2. Orientation of field vectors in a diagonally connected cross-field accelerator

3. Vector diagrams for the crossed-field diagonal MHD devices: (a) Open-circuit generator, (b) $j_{x}$-neutralized generator, (c) short-circuit generator, (d) open-circuit accelerator, (e) zero-efficiency accelerator, and (f) $j_{x}$-neutralized accelerator. Each diagram depicts the relative orientation of the current density vector and electric field vectors based on the generalized Ohm's law, $\frac{\mathbf{j}}{\Sigma}+\Omega\left(\frac{\mathbf{j}}{\Sigma} \times \frac{\mathbf{B}}{B}\right)=\mathbf{E}^{\prime}$, and the generalized electrical field relation, $\mathbf{E}^{\prime}=\mathbf{E}+\mathbf{u} \times \mathbf{B}-\mathbf{E}_{d}$

4. Variation of performance parameters based on representative values for flow/field conditions, material functions, and wall angle. Assumes that $\varphi= \pm 1, B=4 \mathrm{~T}, \Sigma=100$, $\Omega=2, u=3 \mathrm{~km} / \mathrm{s}, \Delta_{O}=-0.1$, and $\Delta_{S}=-0.02$.

5. Performance map for DCW MHD devices with current-dependent voltage drop effect ..... 30

6. Illustration of current transport and boundary layer dimensions for a DCW cross-field accelerator

7. Variation of $\xi_{c}$ and $V_{\Sigma} / 2 \delta$ as a function of $j_{y} / \Sigma_{0}^{\prime}$. Assumes $E_{c}=40 \mathrm{kV} / \mathrm{cm}$, $n=7, m=18$, and $a=10$ 


\section{LIST OF TABLES}

1. Summary of electrical parameter relationships for diagonalized cross-field MHD devices ..

2. Summary of mean power relationships for diagonalized cross-field MHD devices ....

3. Reference conditions for variable $\Delta$ 


\section{NOMENCLATURE}

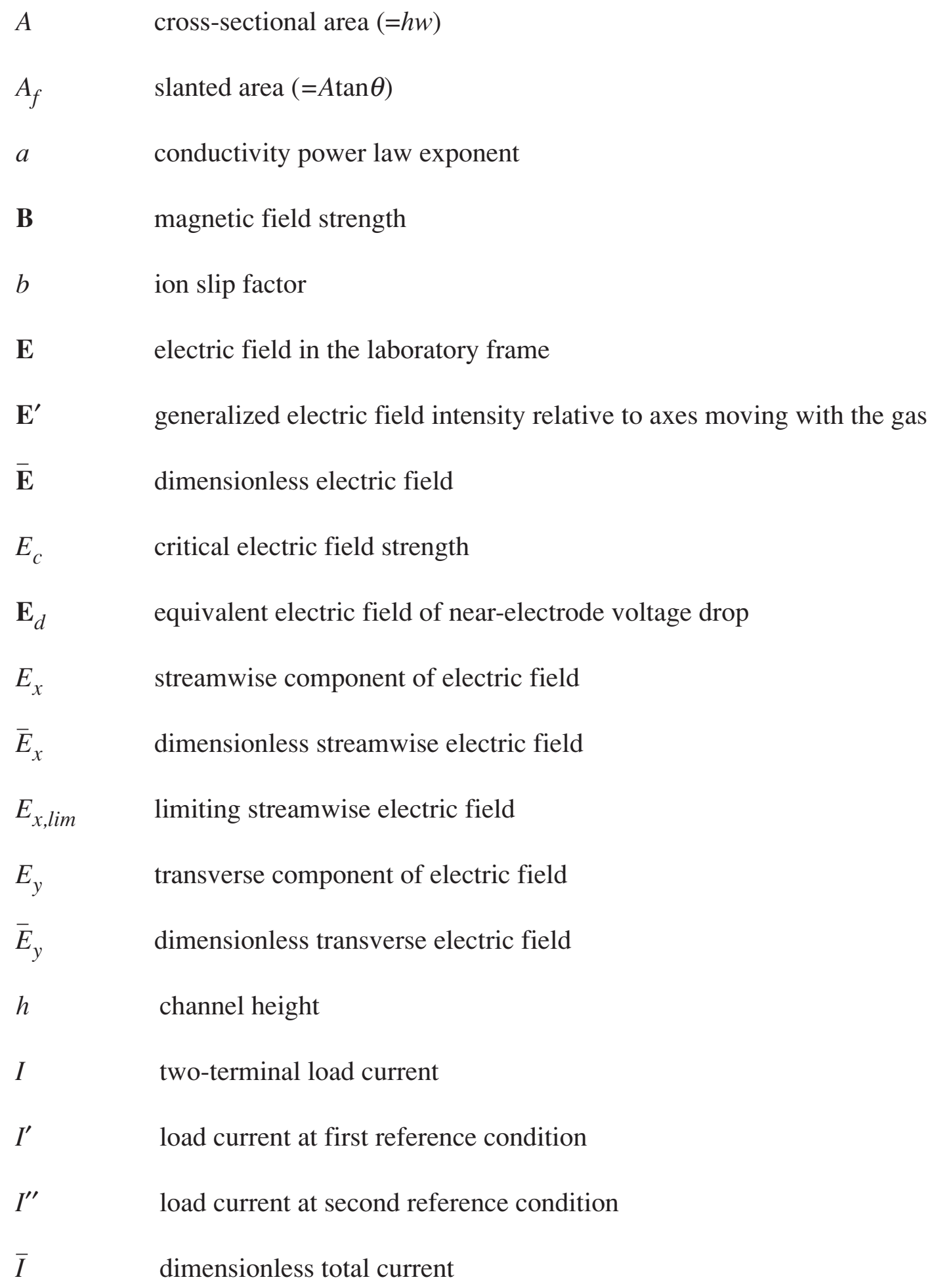




\section{NOMENCLATURE (Continued)}

\begin{tabular}{|c|c|}
\hline$I_{n}$ & load current density for $j_{y}$-neutralized accelerator condition \\
\hline$I_{s}$ & load current for short-circuit generator condition \\
\hline$I_{z}$ & load current for zero-power accelerator condition \\
\hline j & local current density \\
\hline$\overline{\mathbf{j}}$ & dimensionless current density \\
\hline $\bar{j}_{x}$ & dimensionless streamwise current density \\
\hline $\bar{j}_{x, o}$ & open-circuit valve of $\bar{j}_{x}$ \\
\hline $\bar{j}_{x, s}$ & short-circuit valve of $\bar{j}_{x}$ \\
\hline $\bar{j}_{x, \bar{E}=0}$ & valve of $\bar{j}_{x}$ when $\bar{E}_{x}=\bar{E}_{y}=0$ \\
\hline$j_{y}$ & transverse current density \\
\hline $\bar{j}_{y}$ & dimensionless transverse current density \\
\hline $\bar{j}_{y, s}$ & short-circuit valve of $\bar{j}_{y}$ \\
\hline $\bar{j}_{y, \bar{E}=0}$ & valve of $\bar{j}_{y}$ when $\bar{E}_{x}=\bar{E}_{y}=0$ \\
\hline$j_{z}$ & current density along magnetic field lines \\
\hline$L$ & channel length \\
\hline$m$ & thermal boundary layer power law exponent \\
\hline $\mathbf{n}$ & unit vector \\
\hline$n$ & velocity boundary layer power law exponent \\
\hline$P$ & power density \\
\hline $\bar{P}$ & dimensionless power density \\
\hline $\bar{P}_{\max }$ & optimal value of $\bar{P}$ \\
\hline
\end{tabular}




\section{NOMENCLATURE (Continued)}

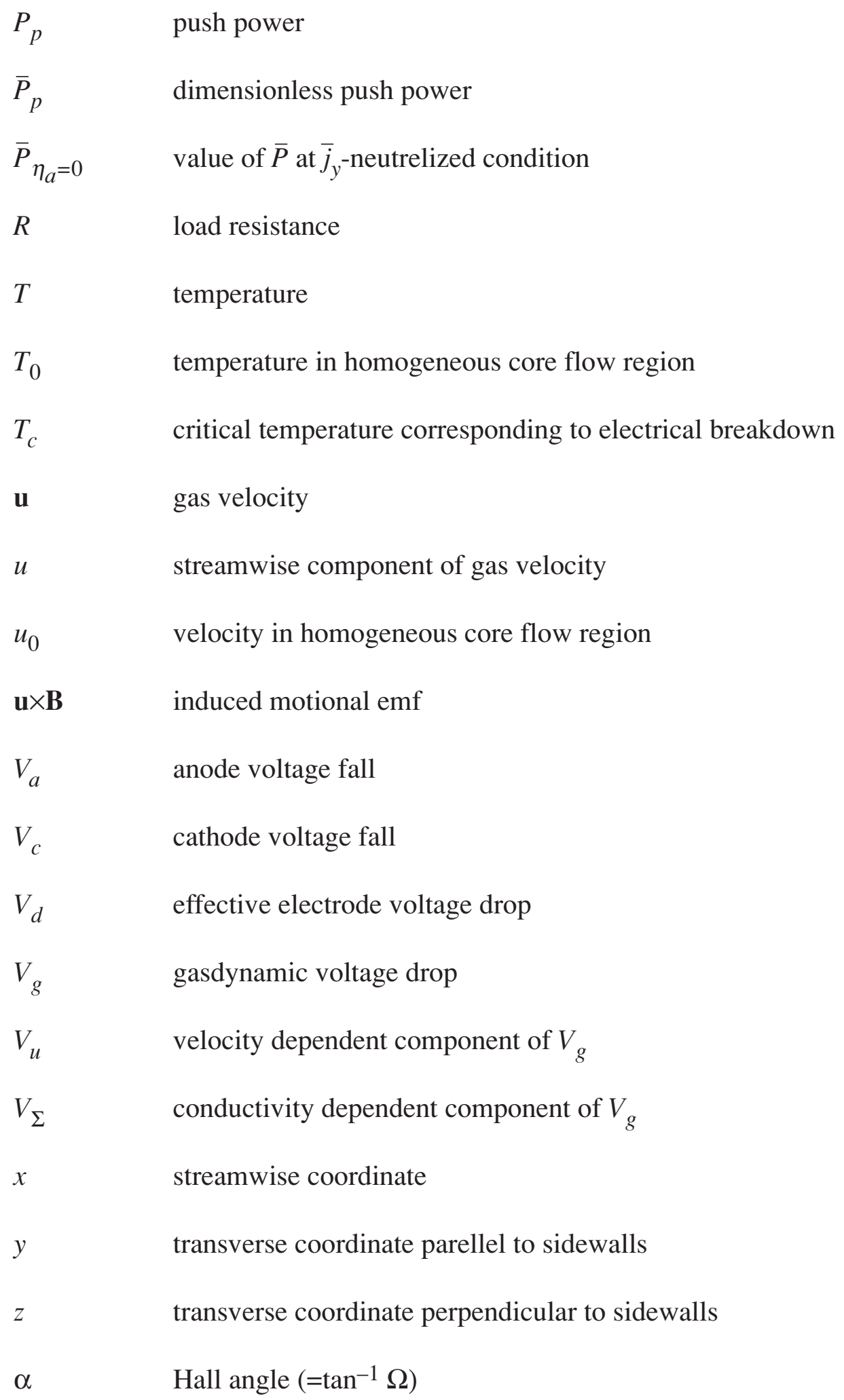




\section{NOMENCLATURE (Continued)}

\begin{tabular}{|c|c|}
\hline$\beta_{e}$ & electron Hall parameter \\
\hline$\beta_{i}$ & ion Hall parameter \\
\hline$\Delta$ & dimensionless voltage drop $\left(=V_{d} / u B h\right)$ \\
\hline$\Delta^{\prime}$ & value of $\Delta$ at first reference condition \\
\hline$\Delta^{\prime \prime}$ & value of $\Delta$ at second reference condition \\
\hline$\Delta_{\max }$ & value of $\Delta$ at maximum power density \\
\hline$\Delta_{n}$ & value of $\Delta$ at $j_{y}$-neutralized condition \\
\hline$\Delta_{o}$ & value of $\Delta$ at open-circuit condition \\
\hline$\Delta_{s}$ & value of $\Delta$ at short-circuit condition \\
\hline$\Delta_{z}$ & value of $\Delta$ at zero-power condition \\
\hline$\delta$ & boundary layer thickness \\
\hline$\delta_{a}$ & anode boundary layer thickness \\
\hline$\delta_{c}$ & cathode boundary layer thickness \\
\hline$\varphi$ & electric field direction \\
\hline$\eta_{a}$ & accelerator electrical efficiency \\
\hline$\eta_{g}$ & generator electrical efficiency \\
\hline$\Sigma$ & effective electrical conductivity \\
\hline$\Sigma_{0}$ & value of $\Sigma$ in homogeneous core flow region \\
\hline$\Sigma_{c}$ & critical value of $\Sigma$ corresponding to electrical breakdown \\
\hline$\sigma$ & scalar electrical conductivity \\
\hline$\theta$ & electric field angle $\left(=\tan ^{-1} \varphi\right)$ \\
\hline
\end{tabular}




\section{NOMENCLATURE (Continued)}

$\theta_{w} \quad$ wall angle or diagonalization

$\Omega \quad$ effective Hall parameter

$\Omega_{0} \quad$ value of $\Omega$ in homogenious core flow region

$\omega \quad$ conductivity ratio

$\omega_{c} \quad$ critical conductivity ratio corresponding to electrical breakdown

$\xi \quad$ dimensionless height parameter $(=y / \delta)$

$\xi_{c} \quad$ critical value of $\xi$ corresponding to electrical breakdown 


\section{TECHNICAL PUBLICATION}

\section{PERFORMANCE THEORY OF DIAGONAL CONDUCTING WALL MAGNETOHYDRODYNAMIC ACCELERATORS}

\section{INTRODUCTION}

Historically, interest in magnetohydrodynamic (MHD) devices has centered on their use as electrical generators in commercial central power plants and mobile burst power systems. The primary attraction for central power is associated with the attainment of higher peak cycle temperatures, which point to significant improvements in overall plant efficiency. The attraction for mobile pulse power, particularly airborne military needs, derives from their intrinsic high power-density characteristics. It should be recognized that equally important though less noted attractions exist for accelerator configurations, as well.

Two major identifiable uses for crossed-field MHD accelerators are (1) propulsive devices and (2) hypersonic aerodynamic test facilities. Litchford et al. discuss the operational attributes that are of particular significance to these applications and provide an indepth historical perspective of their technological development. ${ }^{1}$

From a fundamental point of view, the essential argument favoring the utilization of a Lorentz force acceleration mechanism is the ability to avoid the inherent physical limitations encountered with pure thermal approaches (viz, material thermal limits and ionization/dissociation losses). Simply put, it is more effective to transfer electrical energy into directed kinetic energy rather than first degrading it to thermal energy. Crossed-field acceleration is of special interest in these cases because of its unique capacity for processing large amounts of power under conditions of high mass throughput.

Past emphasis on generator configurations has led to a substantial theoretical and experimental basis for understanding their performance and operational nuances. However, a similar level of understanding has yet to be acquired for accelerator configurations despite a strong growing interest in their potential aerospace applications.

The standard theoretical approach for describing the interaction of an electrically conducting gas with applied electric and magnetic fields relies on the application of a Cowling-Schlueter-type generalized Ohm's law for a partially ionized, electrically neutral gas. Powers et al., for instance, used a cross-plane averaged form of the generalized Ohm's law and developed a graphically based methodology for describing the general performance characteristics of MHD accelerators and generators. ${ }^{2}$

This classical model has proved to be extremely useful in illuminating the basic operational behavior of MHD devices, but its practical utility is hampered by a critical limitation. That is, the theory assumes 
a constant near-electrode voltage drop, whereas experience has shown that the magnitude of the nearelectrode voltage drop exhibits a significant load-current dependency.

Various theoretical refinements of this nature have been forthcoming but solely within the context of generator configurations. Wu, for example, introduced an effective voltage drop parameter, accounted for its load current dependency, and examined the resulting impact on diagonal conducting wall (DCW) generator performance. ${ }^{3}$ The MHD research group at the Max-Planck-Institut fuer Plasmaphysik made a similar attack on the problem using a cross-plane averaged Ohm's law, specialized for a DCW generator configuration. ${ }^{4}$

It is widely recognized, of course, that the current and electric field structure in MHD devices is inherently three-dimensional and that spatial separation of physical processes is not warranted in general. As a matter of recourse, one could mount a brute force attack on the problem and perform a complete threedimensional numerical analysis of the flow and electrical structure in the duct, assuming adequate computing resources are available. Alternatively, one could invoke an infinite segmentation assumption (i.e., the streamwise variation in electrical properties is small in comparison to transverse variations) and couple an approximate cross-plane electrical model with a three-dimensional flow analysis as exemplified for generator configurations by Bityurin et al. and Ahluwalia et al. ${ }^{5,6}$ Each approach has merits and drawbacks, and experience has shown that each level of approximation is useful in fulfilling certain design and analysis needs.

The purpose of this Technical Publication is to reexamine the classical theory and extend it for cross-field accelerator configurations with inclusion of a current-dependent near-electrode voltage drop model. This particular refinement of the classical theory, while generally recognized as a straightforward theoretical extension, has not explicitly appeared in the literature, and it is believed that the resulting development can yield practical insight into the basic operational characteristics of these important devices. Moreover, it is hoped that this work may serve as a convenient and compact resource for future design practitioners. 


\section{CROSSED-FIELD ACCELERATOR CONFIGURATIONS}

Various linear crossed-field MHD accelerator configurations are conceivable (fig. 1) although the optimal configuration depends on the ultimate application need. The Hall configuration, for example, is generally more effective for low-density flows (i.e., very high Hall parameter condition) whereas the segmented Faraday configuration yields superior performance for high-density flows. A significant disadvantage of the Faraday configuration, however, is the separate power conditioning that is required for each electrode pair, which leads to a heavy, complex, and expensive system.

Alternative two-terminal loading schemes have been proposed to avoid the complications of multiterminal connections while also attempting to compensate for the effects associated with a finite Hall parameter. De Montardy, for instance, suggested the series connected scheme in which a segmented Faraday channel is externally diagonalized. ${ }^{7}$ Dicks later extended this approach to DCW configuration in which slanted window frame-like electrode elements are stacked with thin insulators to form a complete channel. ${ }^{8,9}$ The DCW configuration not only simplifies fabrication and improves strength but provides superior performance to the series connected device by allowing current to flow to the sidewalls. ${ }^{3}$

Hence, theoretical development is focused on the DCW accelerator configuration, since it has the greatest practical relevance. The performance characteristics of the alternative crossed-field configurations can be subsequently deduced as special or extreme cases of the DCW configuration. 


\section{GENERALIZED OHM'S LAW}

The local current density, $\mathbf{j}$, in a partially ionized, electrically neutral gas may be determined from a Cowling-Schlueter-type generalized Ohm's law. ${ }^{10-14}$ For application to typical MHD devices, it is useful to adopt the simplified form derived by Rosa, which includes ion slip but neglects electron pressure gradients: ${ }^{15}$

$$
\mathbf{j}=\sigma \mathbf{E}^{\prime}-\frac{\beta_{e}}{B} \mathbf{j} \times \mathbf{B}+\frac{\beta_{e} \beta_{i}}{B^{2}}(\mathbf{j} \times \mathbf{B}) \times \mathbf{B} .
$$

Here, $\sigma$ is the scalar electrical conductivity; $\mathbf{E}^{\prime}$ is the generalized electric field intensity relative to axes moving with gas velocity, $\mathbf{u} ; B$ is the magnetic field strength; and $\beta_{e}$ and $\beta_{i}$ are the electron and ion Hall parameters, respectively. The Hall effect and ion slip are introduced by the second and third terms on the right-hand side of equation (1).

As a practical matter, we define the generalized electric field, $\mathbf{E}^{\prime}$, in terms of the electric field in the laboratory frame, $\mathbf{E}$; the motional emf, $\mathbf{u} \times \mathbf{B}$; and an equivalent electric field, $\mathbf{E}_{d}$, which accounts for the near-electrode voltage drop, $V_{d}$ (i.e., $E_{d}=V_{d} / h$ ), inside the boundary layer,

$$
\mathbf{E}^{\prime}=\mathbf{E}+\mathbf{u} \times \mathbf{B}-\mathbf{E}_{d} .
$$

Note that the equivalent electric field is applied only in a direction transverse to the magnetic field vector and the streamwise velocity vector. It is always directed in opposition to the transverse current, $j_{y}$.

For the special case in which no current flows along the magnetic field lines, $\left(j_{z}=0\right)$, it is readily shown that equation (1) reduces to the compact form

$$
\mathbf{j}=\Sigma \mathbf{E}^{\prime}-\frac{\Omega}{B} \mathbf{j} \times \mathbf{B},
$$

where we have introduced two new parameters:

$$
\Sigma=\frac{\sigma}{1+\beta_{e} \beta_{i}}=\frac{\sigma}{1+b}
$$

and

$$
\Omega=\frac{\beta_{e}}{1+\beta_{e} \beta_{i}}=\frac{\beta_{e}}{1+b} .
$$


In many cases, the ion slip factor, $b=\beta_{e} \beta_{i}$, is negligible, and further simplification is obtained as $\Sigma \rightarrow \sigma$ and $\Omega \rightarrow \beta_{e}$.

\subsection{Mean Ohm's Law}

Many of the interesting phenomena that occur in high-interaction MHD devices, such as velocity overshoots in the sidewall boundary layer, flow asymmetries, and generation of intense secondary flows in the cross plane, are three-dimensional in nature. Therefore, high-fidelity performance predictions ultimately require three-dimensional numerical analyses of the combined flow and electrical structure. On the other hand, simplified approaches based on spatial decoupling or reduced spatial dimensions can also yield useful results if all critical physical effects are accounted for in an appropriate fashion.

Exact theoretical treatment requires consideration of the fundamental equations of MHD and Maxwell's electromagnetic equations, thereby leading to a system of coupled differential equations. Here, we invoke the infinite segmentation assumption and assume that all parameters in the generalized Ohm's law can be effectively represented by their cross-plane averaged values and are dependent on the $x$-coordinate, only.

The dimensionless voltage drop parameter, $\Delta=E_{d} / u B=V_{d} / u B h$, which incorporates all voltage losses associated with the electrode boundary layer, may be introduced into the generalized Ohm's law to obtain the component relations,

$$
j_{x}=\Sigma E_{x}-\Omega j_{y}
$$

and

$$
j_{y}=\Sigma E_{y}-\Sigma u B(1+\Delta)+\Omega j_{x}
$$

Equations (6) and (7) may also be combined to obtain the inverse expressions for $j_{x}$ and $j_{y}$ in terms of the local electric field components,

$$
j_{x}=\frac{\Sigma}{1+\Omega^{2}}\left[E_{x}-\Omega E_{y}\right]+\frac{\Omega}{1+\Omega^{2}} \Sigma u B(1+\Delta)
$$

and

$$
j_{y}=\frac{\Sigma}{1+\Omega^{2}}\left[\Omega E_{x}+E_{y}\right]-\frac{1}{1+\Omega^{2}} \Sigma u B(1+\Delta) .
$$




\section{PERFORMANCE MODEL}

\subsection{Diagonal Loading Constraint}

Completion of the electrical model requires the consideration of external loading conditions. In the case of a diagonally linked device (fig. 2), the electric field is forced to align perpendicularly to the diagonal short so that

$$
E_{y} / E_{x}=\tan \theta=\varphi
$$

where $\theta$ is the electric field angle and $\varphi$ is the electric field direction. It follows that the diagonalization or wall angle is given by $\theta_{w}=\pi / 2-\theta$. The sign of $\varphi$ depends on the mode of operation. For an accelerator, $\mathbf{u} \times \mathbf{B}$ opposes $j_{y}, 0<\theta<\pi / 2$, and $\varphi>0$. For a generator, $\mathbf{u} \times \mathbf{B}$ aligns with $j_{y},-\pi / 2<\theta<0$, and $\varphi<0$.

The two-terminal load current, $I$, for an MHD device with diagonally linked electrode pairs is given by

$$
I=\int_{A_{f}} \mathbf{j} \cdot d \mathbf{A}_{f}=\mathbf{j} \cdot \mathbf{n} A_{f}
$$

where the integration is over the entire slanted area, $A_{f}$. In component form,

$$
I=\left(j_{x}+\tan \theta j_{y}\right) A=\left(j_{x}+\varphi j_{y}\right) A
$$

\subsection{Electrical Parameter Relationships}

A complete set of equations now exists for determining the cross-plane electrical characteristics. In general, either $I$ or $E_{x}$ may be specified, allowing the remaining unknown electrical parameters to be deduced from equations (6), (7), (10), and (12) in conjunction with appropriate material functions.

The resulting performance relationships for DCW accelerators are summarized in table 1 using $E_{x}$ and $I$ as independent variables. The governing relations for generator performance are recovered when both $\varphi$ and $\Delta$ are less than zero and $I=-E_{x} L / R$, where $R$ is the load resistance and $L$ is the channel length over which the load is applied. Note also that the governing relations for a linear Hall channel configuration are recovered in the extreme case $\varphi=0$. 


\subsection{Mean Power Parameters}

Ultimately, we seek to deduce suitably averaged power parameters from the resulting cross-plane electrical model. The quantities $\mathbf{j} \cdot \mathbf{E}$ and $(\mathbf{j} \times \mathbf{B})_{x}$, for instance, may be used to determine the power density, push power, dissipated power, and electrical efficiency of a device.

\subsubsection{Power Density}

The electrical power density at any cross section of the duct is defined by

$$
P=\mathbf{j} \cdot \mathbf{E}=\frac{I}{A_{f}} \sqrt{E_{x}^{2}+E_{y}^{2}}
$$

Eliminating $E_{y}$ using equation (10) yields

$$
P=\frac{I}{A_{f}} \sqrt{E_{x}^{2}+\varphi^{2} E_{x}^{2}}=\frac{I E_{x}}{A_{f}} \sqrt{1+\varphi^{2}}
$$

but given $A_{f}=A \sqrt{1+\varphi^{2}}$, the working form becomes

$$
P=\frac{I E_{x}}{A}
$$

\subsubsection{Push Power}

The streamwise Lorentz body force component at any cross section is defined as

$$
(\mathbf{j} \times \mathbf{B})_{x}=j_{y} B
$$

and the push power associated with this Lorentz body force is given by

$$
P_{p}=\mathbf{u} \cdot(\mathbf{j} \times \mathbf{B})_{x}=u j_{y} B
$$

\subsubsection{Dissipated Power}

The power density of an MHD device must exceed the Lorentz force push power since the internal resistance to current flow leads to ohmic heating. This can be demonstrated by taking the dot product of $\mathbf{j}$ with the generalized Ohm's law, as defined by equation (3), which yields 


$$
\mathbf{j} \cdot \mathbf{j}=\Sigma\left[\mathbf{j} \cdot \mathbf{E}+\mathbf{j} \cdot(\mathbf{u} \times \mathbf{B})-\mathbf{j} \cdot \mathbf{E}_{d}\right]-\frac{\Omega}{B} \mathbf{j} \cdot(\mathbf{j} \times \mathbf{B})
$$

Because $j_{z}=0$, the last term is identically zero, and we obtain

$$
\frac{\mathbf{j} \cdot \mathbf{j}}{\Sigma}=\mathbf{j} \cdot \mathbf{E}-\mathbf{u} \cdot \mathbf{j} \times \mathbf{B}-\mathbf{j} \cdot \mathbf{E}_{d}=P-P_{p}-j_{y} u B \Delta
$$

where $\mathbf{j} \cdot \mathbf{j} / \Sigma$ is the power dissipated in the core flow region, and $\mathbf{j} \cdot \mathbf{E}_{d}=j_{y} E_{d}=j_{y} u B \Delta$ represents the power dissipated in the electrical boundary layers. Therefore, the power dissipated over the entire cross plane is given by

$$
P_{d}=\frac{\mathbf{j} \cdot \mathbf{j}}{\Sigma}+j_{y} u B \Delta=P-P_{p}
$$

\subsubsection{Electrical Efficiency}

The electrical efficiency of an accelerator is simply the ratio of the push power to the applied power:

$$
\eta_{a}=\frac{P_{p}}{P}=\frac{\mathbf{u} \cdot(\mathbf{j} \times \mathbf{B})_{x}}{\mathbf{j} \cdot \mathbf{E}}=\frac{u j_{y} B}{I E_{x} / A} .
$$

It follows that the generator efficiency is defined as the reciprocal of the accelerator efficiency, $\eta_{g}=1 / \eta_{a}$.

\subsubsection{Power Relationships}

By combining the results in table 1 with the relationships above, it is possible to express the mean power parameters in terms of the independent variables $E_{x}$ or $I$. These results are summarized in table 2.

\subsection{Generalized Vector Diagram}

It is generally instructive to examine the qualitative behavior of the internal electric fields and current densities under the assumption that MHD effects have only a slight effect on flow properties. In this case, the generalized Ohm's law can be used to construct representative vector diagrams for diagonalized devices, as shown in figure 3 for fixed values of $\varphi$.

To construct these diagrams, the generalized Ohm's law defined by equation (3) is put into the form

$$
\frac{\mathbf{j}}{\Sigma}+\Omega\left(\frac{\mathbf{j}}{\Sigma} \times \frac{\mathbf{B}}{B}\right)=\mathbf{E}^{\prime},
$$


where we immediately recognize that the two terms on the left-hand side of equation (22) must be orthogonal. Thus, each of these terms represents one side of a right triangle with the hypotenuse, $\mathbf{E}^{\prime}$, inclined at an angle of $\tan ^{-1} \Omega$. The generalized electric field must simultaneously satisfy the vector relation defined by equation (2).

Figures 3(a)-(c) illustrate the load current dependence of a diagonalized generator $(\varphi<0)$. In the open-circuit case (a), there is no current flow to the load, and the internal current density vector must align with the diagonal link, which defines planes of constant electric potential. Thus, the current is closed through the diagonal short, and $\mathbf{E}_{y}$ exactly compensates for the combined $\mathbf{u} \times \mathbf{B}$ and $\mathbf{E}_{d}$ induced potentials. Note that $j_{x}$ is directed upstream under these conditions. As the load current is increased, the current density vector rotates out of the plane of the diagonal link until it aligns with the $\mathbf{u} \times \mathbf{B}$ vector. At this point, $j_{x}=0$ and we arrive at the Hall current neutralized condition (b). If the load current is increased further, the current vector continues its rotation, and the direction of Hall current flow is reversed. Ultimately, the current density vector becomes perpendicular to the plane of the diagonal link as the electric field goes to zero at the short-circuit condition (c).

Figures 3(d)-(f) illustrate the load current dependence of a diagonalized accelerator $(\varphi>0)$. When the applied axial electric field is zero and there is no load current, the open-circuit accelerator loading condition is obtained $(\mathrm{d})$. The internal current density vector corresponds to the generator short-circuit case, under these unique circumstances. Note that $j_{y}<0$ (braking regime) and that the current flow is closed through the diagonal short. With increasing applied axial electric field, the current density vector rotates out of the plane of the diagonal link. The device remains in a braking regime, however, until the applied field becomes large enough to force $j_{y}$ to zero at which point we attain the zero-efficiency accelerator condition (e). Further increases in applied current lead to continued rotation of the current density vector and increasing values of $j_{y}$. Eventually, one reaches a $j_{x}$ neutralized-accelerator condition (f). Increasing the applied current beyond this point leads to a reversal in the direction of axial current flow while the magnitude of the current density vector continues to grow.

\subsection{Current-Dependent Voltage Drop}

The previously developed performance relationships hold for any fixed value of $\Delta$. However, extensive experimental research with MHD generators has clearly established that the near-electrode volt-

age drop is approximately linearly dependent on the transverse current density. ${ }^{16-18}$ That is, any increase in load current leads to a proportionate increase in voltage drop. This behavior is assumed to extend to accelerator operation, as well.

The linear relationship between $V_{d}$ and $j_{y}$ permits the construction of a simple two-parameter model,

$$
V_{d}=\left(\frac{V_{d}^{\prime \prime}-V_{d}^{\prime}}{I^{\prime \prime}-I^{\prime}}\right)\left(I-I^{\prime}\right)+V_{d}^{\prime},
$$

where the prime and double-prime superscripts indicate two distinct loading conditions. It is generally more convenient to express this relation directly in terms of $\Delta$ : 


$$
\Delta=\left(\frac{\Delta^{\prime \prime}-\Delta^{\prime}}{I^{\prime \prime}-I^{\prime}}\right)\left(I-I^{\prime}\right)+\Delta^{\prime}
$$

Selection of appropriate reference load conditions depends on the mode of operation. For a generator, it is most natural to adopt the open-circuit $\left(\Delta^{\prime}=\Delta_{O}\right)$ and short-circuit $\left(\Delta^{\prime \prime}=\Delta_{S}\right)$ conditions on the extreme ends of the operational load line. For an accelerator, it is convenient to adopt the zero-power condition, which corresponds to a short-circuit generator $\left(\Delta^{\prime}=\Delta_{z}=\Delta_{s}\right)$, and the $j_{y}$ neutralized condition $\left(\Delta^{\prime \prime}=\Delta_{n}=0\right)$. The selected reference conditions are summarized in table 3. The implications of this model are now examined for the generator and accelerator modes.

\subsubsection{Generator Mode}

At the short-circuit generator condition, $E_{x}=0, I^{\prime \prime}=I_{S}$, and $\Delta^{\prime \prime}=\Delta_{S}$. From table 1 , we therefore deduce

$$
I^{\prime \prime}=I_{S}=\frac{\sum u B A\left(1+\Delta_{S}\right)(\Omega-\varphi)}{1+\Omega^{2}}
$$

Elimination of $I^{\prime \prime}$ using equation (24) immediately yields an expression for $\Delta$ in terms of $I$ for a generator:

$$
\Delta=\Delta_{O}-\left[\frac{\left(1+\Omega^{2}\right)\left(\Delta_{o}-\Delta_{S}\right)}{(\Omega-\varphi)\left(1+\Delta_{S}\right)}\right] \frac{I}{\Sigma u B A} .
$$

This relationship may be used to eliminate $\Delta$ from the performance relations of tables 1 and 2 .

\subsubsection{Accelerator Mode}

The zero-power open-circuit accelerator condition corresponds to the short-circuit generator condition with $\varphi>0$. In this case, $E_{x}=0, I^{\prime}=I_{z}$, and $\Delta^{\prime}=\Delta_{z}=\Delta_{S}$, and we deduce that

$$
I^{\prime}=I_{z}=\frac{\sum u B A\left(1+\Delta_{S}\right)(\Omega-\varphi)}{1+\Omega^{2}} .
$$

The point of transverse current-density neutralization corresponds to a zero-efficient accelerator where $j_{y}=0, I^{\prime \prime}=I_{n}$, and $\Delta^{\prime \prime}=\Delta_{n}=0$. This implies

$$
I^{\prime \prime}=I_{n}=\frac{\Sigma u B A}{\Omega+\varphi}
$$


Eliminating $I^{\prime}$ and $I^{\prime \prime}$ in equation (24) gives an expression for $\Delta$ in terms of $I$ for an accelerator,

$$
\Delta=\Delta_{S}\left[1-\frac{(\Omega+\varphi)\left(1+\Omega^{2}\right) \frac{I}{\Sigma u B A}-\left(\Omega^{2}-\varphi^{2}\right)\left(1+\Delta_{S}\right)}{\left(1+\Omega^{2}\right)-\left(\Omega^{2}-\varphi^{2}\right)\left(1+\Delta_{S}\right)}\right] .
$$

This relationship may be used to eliminate $\Delta$ in the performance relations of tables 1 and 2 . 


\section{PERFORMANCE DIAGRAMS}

It is possible to construct an informative performance map for DCW MHD devices following the general methodology first outlined by Powers et al., who assumed a constant voltage drop in the electrode sheath layer. ${ }^{2}$ The significant modifications associated with a variable effective voltage drop model were

later noted and described by Wu for the DCW generator case. ${ }^{3}$ Here, Wu's analysis is explicitly extended for DCW accelerator operation, as well.

\subsection{Dimensionless Performance Parameters}

As a first step, the governing performance relationships are simplified by defining the following dimensionless quantities:

$$
\bar{j}=\frac{\mathbf{j}}{\Sigma u B}, \bar{E}=\frac{\mathbf{E}}{u B}, \bar{I}=\frac{I}{\Sigma u B A}, \bar{P}=\frac{P}{\Sigma u^{2} B^{2}} .
$$

Substitution into the component form of the generalized Ohm's law then yields

$$
\bar{j}_{x}=\frac{1}{1+\Omega^{2}}\left[\bar{E}_{x}+\Omega \bar{E}_{y}+\Omega(1+\Delta)\right]
$$

and

$$
\bar{j}_{y}=\frac{1}{1+\Omega^{2}}\left[\Omega \bar{E}_{x}+\bar{E}_{y}-(1+\Delta)\right] .
$$

It also follows that the electric field components can be stated explicitly in terms of current density:

$$
\bar{E}_{x}=\bar{j}_{x}+\Omega \bar{j}_{y}
$$

and

$$
\bar{E}_{y}=-\Omega \bar{j}_{x}+\bar{j}_{y}+(1+\Delta)
$$

An expression for the dimensionless load current is obtained from the substitution of equation (30) into equation (12):

$$
\bar{I}=\bar{j}_{x}+\varphi \bar{j}_{y}
$$


and the dimensionless laboratory power density follows directly from the substitution of equation (30) into equation (13):

$$
\bar{P}=\overline{\boldsymbol{j}} \cdot \overline{\boldsymbol{E}}=\bar{j}_{x} \bar{E}_{x}+\bar{j}_{y} \bar{E}_{y}
$$

Eliminating $\bar{E}_{x}$ and $\bar{E}_{y}$ using equations (33) and (34) yields the more convenient form,

$$
\bar{P}=\bar{j}_{x}^{2}+\bar{j}_{y}^{2}+(1+\Delta) \bar{j}_{y}
$$

Furthermore, we may define the dimensionless push power as

$$
\bar{P}_{p}=\frac{P_{p}}{\Sigma u^{2} B^{2}}=\frac{u j_{y} B}{\Sigma u^{2} B^{2}}=\frac{j_{y}}{\Sigma u B}=\bar{j}_{y},
$$

and the electrical efficiency may be expressed in terms of dimensionless parameters by combining equations (37) and (38):

$$
\eta_{a}=\frac{\bar{P}_{p}}{\bar{P}}=\frac{\bar{j}_{y}}{\bar{j}_{x}^{2}+\bar{j}_{y}^{2}+(1+\Delta) \bar{j}_{y}}=\frac{1}{\eta_{g}}
$$

It is readily apparent that the relationships in tables 1 and 2 can all be put in dimensionless form using equation (30); the results are obvious and are not tabulated. The current-dependent effective voltage drop effect is accounted for by using either equation (26) or (29) in dimensionless form.

As a prelude to the construction of the graphical performance map, it is instructive to examine performance parameter variations as a function of the dimensionless axial electric field, $\bar{E}_{x}$. Figure 4 , for example, summarizes calculated parameter variations assuming representative values for electrical/flow conditions, material functions, and wall angle. Starting from the open-circuit generator condition and gradually reducing the load impedance, we observe the sequential occurrence of all of the loading conditions depicted in figure 3 .

For an open-circuit generator, $\bar{I}=0$ and equation (35) yield the constraint $\bar{j}_{x, o}=-\varphi \bar{j}_{x, O}$. We therefore infer that $\bar{j}_{x, o}<0$ since $\varphi<0$ and $\bar{j}_{y, o}<0$ for the assumed magnetic field direction. In this case, the internal current is forced parallel to the wall angle and is completely shorted through the diagonal linkage. As the load impedance is decreased below a critical level, however, the total current becomes nonzero, and the magnitude of the negatively directed axial electric field begins to fall.

If the load impedance is decreased all the way to zero, we arrive at the short-circuit generator condition corresponding to $\bar{E}_{x}=0$. Here, application of equation (33) yields the constraint $\bar{j}_{x, s}=-\Omega \bar{j}_{y, s}$, which implies $\bar{j}_{x, s}>0$ since $\Omega>0$ and $\bar{j}_{y, s}<0$. In this case, the internal current flow is forced perpendicular to the wall angle, and no current flows through the diagonal linkage. 
Consideration of these open- and short-circuit constraints clearly implies the existence of a Hall current neutralized condition (i.e., $\bar{j}_{x}=0$ ) on the generator load line. It is also clear that maximum power extraction and electrical efficiency must also occur at some point between the open- and short-circuit conditions. This point may or may not coincide with the Hall current neutralized condition. Note that the magnitude of $\Delta$ is largest at open-circuit loading where the magnitude of $\bar{j}_{y}$ is greatest.

Simply reversing the wall angle $(\varphi>0)$ at the generator short-circuit condition yields the opencircuit accelerator condition. If power is then gradually applied such that a positive axial electric field is impressed on the accelerator, we enter a regime where the transverse voltage is insufficient to overcome the induced $\mathbf{u} \times \mathbf{B}$ potential, and $\bar{j}_{y}$ remains negatively directed. This is commonly referred to as the braking regime, where all applied power goes into joule heating of the working fluid. Eventually, the applied voltage becomes high enough to neutralize the transverse current at which point $\Delta \rightarrow 0$.

Increasing the applied electric field beyond this point yields positive push work and flow acceleration. The transverse current and total current grow in magnitude, more power is delivered to the device, and $\Delta$ steadily increases. At the same time, axial current flow gradually decreases until the $\bar{j}_{x}$ neutralization condition is reached. Additional increases in applied field and power beyond this point simply reverse the direction of $\bar{j}_{x}$. It is important to note that optimal accelerator electrical efficiency occurs at an applied electric field magnitude much less than that required to neutralize axial current flow.

\subsection{Performance Map}

The operational attributes of MHD devices can be exhibited in the most vivid manner on a performance map in the plane of dimensionless current density and electric field. Following the methodology of Powers et al., ${ }^{2}$ as it was modified by $\mathrm{Wu}$ for current-dependent voltage drop, ${ }^{3}$ we complete the square in equation (37) to obtain the family of circles defined by

$$
\bar{j}_{x}^{2}+\left[\bar{j}_{y}+\frac{1+\Delta}{2}\right]^{2}=\bar{P}+\left(\frac{1+\Delta}{2}\right)^{2} .
$$

These circles are centered at $\bar{j}_{x}=0, \bar{j}_{y}=(1+\Delta) / 2$ with a radius of $\left\{\bar{P}+[(1+\Delta) / 2]^{2}\right\}^{1 / 2}$.

Transformation to the $\bar{E}_{x}, \bar{E}_{y}$ axes is defined by equations (33) and (34), and the origin of the $\bar{E}_{x}, \bar{E}_{y}$ axes in the $\bar{j}_{x}, \bar{j}_{y}$ plane follows directly from equations (31) and (32):

$$
\bar{j}_{x, \bar{E}=0}=\frac{\Omega(1+\Delta)}{1+\Omega^{2}} \quad \bar{j}_{y, \bar{E}=0}=-\frac{(1+\Delta)}{1+\Omega^{2}} .
$$

The locus of points defining this origin can be expressed independently of $\Omega$ by combining equations (33) and (34) under the condition $\bar{E}_{x}=\bar{E}_{y}=0$ :

$$
\bar{j}_{x, \bar{E}=0^{+}}^{2}\left[\bar{j}_{y, \bar{E}=0}+\frac{1+\Delta}{2}\right]^{2}=\left(\frac{1+\Delta}{2}\right)^{2} .
$$


Note that the circles defined by equation (40) reduce to the loci defined by equation (42) at zero-power density. Thus, the generator boundary coincides with the locus of the origin of the $\bar{E}_{x}, \bar{E}_{y}$ axes, and all power generating circles lie inside this boundary since $\bar{P}<0$ for a generator. Further insight may be gained through the examination of extreme generator loading conditions.

At generator short circuit, the Hall field goes to zero, and equation (33) gives the constraint

$$
\bar{j}_{x, s}=-\Omega \bar{j}_{y, s} \Rightarrow \tan ^{-1}(-1 / \Omega)=\bar{j}_{y, s} / \bar{j}_{x, s}
$$

Because $\Omega>0$ and $\bar{j}_{y}<0$, given the assumed magnetic field orientation, the short-circuit operating domain resides in the fourth quadrant of the current density plane. constraint

At generator open-circuit conditions, the total current falls to zero and equation (35) gives the

$$
\bar{j}_{x, o}=-\varphi \bar{j}_{y, o} \Rightarrow \tan ^{-1}(-1 / \varphi)=\bar{j}_{y, o} / \bar{j}_{x, o}
$$

Because $\varphi<0$ and $\bar{j}_{y}<0$ for the generator mode, the open-circuit operating domain resides in the third quadrant of the current density plane.

Clearly, the power density vanishes at both open and short circuit conditions, but the critical discriminating factor to note is that $\Delta$ varies with current over the entire load range $\left(-1<\Delta_{O}<\Delta_{S}<0\right)$. Therefore, the radius of the zero-power circle depends on the load. The zero-power loci for short- and opencircuit loading follows from equation (40) with $\bar{P}=0$ :

$$
\bar{j}_{x, s}^{2}+\left[\bar{j}_{y, s}+\frac{1+\Delta_{s}}{2}\right]^{2}=\left(\frac{1+\Delta_{s}}{2}\right)^{2}
$$

and

$$
\bar{j}_{x, o}^{2}+\left[\bar{j}_{y, o}+\frac{1+\Delta_{O}}{2}\right]^{2}=\left(\frac{1+\Delta_{O}}{2}\right)^{2} .
$$

Using these expressions, it is possible to construct the short- and open-circuit half-circles. The short-circuit half-circle is centered at $\bar{j}_{x}=0, \bar{j}_{y}=\left(1+\Delta_{s}\right) / 2$ with radius $\left(1+\Delta_{s}\right) / 2$, and the open-circuit halfcircle is centered at $\bar{j}_{x}=0, \bar{j}_{y}=\left(1+\Delta_{o}\right) / 2$ with radius $\left(1+\Delta_{o}\right) / 2$. These loci are illustrated in figure 5 where the load line connects in accordance with the constraints defined by equations (43) and (44). Because $\Delta_{O}<\Delta_{S}$, the radius of the open-circuit half-circle is smaller than the radius of the short-circuit half-circle, and the center points are shifted.

The origin of the $\bar{E}_{x}, \bar{E}_{y}$ axes falls on the short-circuit half-circle at the points defined by equation (41). Inspection of equation (34) indicates that the $\bar{E}_{y}$ axis passes through the origin of the $\bar{j}_{x}, \bar{j}_{y}$ axes since $\Delta=0$ at this condition. The various points of interest are indicated in figure 5 . 
Combining the DCW electric field constraint defined by equation (10) with equations (30), (33), and (34) yields an expression for the operating load line in the form

$$
(\Omega+\varphi) \bar{j}_{x}+(\Omega \varphi-1) \bar{j}_{y}=1+\Delta
$$

It is now possible to eliminate $\Delta$ using either equation (26) for a generator or equation (29) for an accelerator. In both cases, one ends up with an equation for a line in the $\bar{j}_{x}, \bar{j}_{y}$ plane since $\Delta$ is linearly related to $\bar{j}_{x}$ and $\bar{j}_{y}$. The resulting operating lines are illustrated in figure 5 .

In the generator case, the operating line passes through the short- and open-circuit load conditions, as determined by equations (43) and (44), respectively. In the accelerator case, the operating line originates from the generator short-circuit loading point on the zero-power half-circle. This line also projects through a point on the half-circle defined by the value of $\varphi$.

The $\bar{P}$ circles for accelerator operation are obtained from equation (40) using equation (29) to eliminate $\Delta$. Note that the accelerator operates in the dissipative braking regime between open-circuit and zero-efficiency conditions. Thus, positive push work is obtained only when the power density exceeds that associated with the zero-efficiency condition where $\bar{j}_{y}=\eta_{a}=\Delta_{n}=0$ so that

$$
\bar{P}_{\eta_{a}=0}=\bar{j}_{x} \bar{E}_{x}=\left(\frac{1+\Delta_{n}}{\Omega+\varphi}\right)^{2}=\frac{1}{(\Omega+\varphi)^{2}} .
$$

Portions of the constant $\bar{P}$ circles lying within the positive push work regime are illustrated in figure 5. Inspection of equation (40) reveals that the radii of these circles are larger than those that would occur for an ideal device in which there is no parasitic voltage drop $(\Delta=0)$. Thus, additional power must be applied in a real device to achieve a desired intensity of acceleration.

It is important to note that the optimum accelerator efficiency occurs with a finite axial current. Increasing the accelerator power density until $\bar{j}_{x}$ vanishes will inevitably reduce the electrical efficiency below the optimal value. In fact, to accelerate plasma efficiently (with little heat production), it is desirable to maintain gentle acceleration levels by keeping the back emf only slightly less than the applied electric field. In practice, the added length and weight associated with gentle acceleration must be traded against the electrical inefficiencies encountered with high push power.

For a generator, the maximum power condition can differ considerably from the maximum efficiency condition. Furthermore, maximum generator efficiency operation also occurs with a finite axial current as observed in figure 4. It should be pointed out that achieving optimal power generation for a given value of Hall parameter depends on careful selection of the wall angle. This is examined for the sake of completeness.

By definition, the dimensionless power density may be expressed in the form

$$
\bar{P}=\frac{P}{\Sigma u^{2} B^{2}}=\frac{I E_{x} / A}{\Sigma u^{2} B^{2}}=\bar{I} \bar{E}_{x}=\left(\bar{j}_{x}+\varphi \bar{j}_{y}\right) \bar{E}_{x} .
$$


If we now use equation (26) to eliminate $\bar{I}$ in the appropriate expressions for $\bar{j}_{x}, \bar{j}_{y}$, and $\bar{E}_{x}$, we obtain the dimensionless generator power density in terms of $\Delta$

$$
\bar{P}=\frac{\left(\Omega-\varphi^{2}\right)\left(1+\Delta_{S}\right)^{2}}{\left(1+\varphi^{2}\right)\left(1+\Omega^{2}\right)}\left(\frac{\Delta-\Delta_{O}}{\Delta_{S}-\Delta_{o}}\right)\left[\frac{\Delta-\Delta_{O}}{\Delta_{S}-\Delta_{o}}-\frac{1+\Delta}{1+\Delta_{s}}\right]
$$

The maximum power density is defined by the constraint $\partial \bar{P} / \partial \Delta=0$, which yields the relation

$$
\Delta_{\max }=\frac{\Delta_{O}+\Delta_{S}}{2}
$$

Substituting this result into equation (50) then yields

$$
\bar{P}_{\max }=\frac{-\left(\Omega-\varphi^{2}\right)}{\left(1+\varphi^{2}\right)\left(1+\Omega^{2}\right)} \frac{\left(1+\Delta_{S}\right)\left(1+\Delta_{o}\right)}{4}
$$

Thus, $\bar{P}_{\text {max }}$ depends on $\varphi$, and the optimal wall angle for a given Hall parameter is determined by the constraint $\partial \bar{P}_{\max } / \partial \varphi=0$, which leads to the design criterion,

$$
\Omega \varphi=-1
$$

If this criterion is enforced, we find the maximum possible generator power density to be

$$
\bar{P} \max (\Omega \varphi=-1)=-\frac{\left(1+\Delta_{S}\right)\left(1+\Delta_{o}\right)}{4} .
$$




\section{ELECTRODE BOUNDARY LAYER EFFECTS}

The effective voltage drop arises from cold-wall boundary layer effects including gasdynamic variations, discharge constriction, and electrode falls. Because fluid temperature and velocity vary rapidly approaching the wall, the conductivity and induced voltage also exhibit strong variations in the thermal and momentum boundary layers, respectively. Furthermore, the rapid decrease in temperature near the electrode surface leads to breakdown and a sudden switch from diffuse current transport to constricted arcs. Current attachment to cold electrodes almost always occurs through a thin layer of short arcs.

The basic boundary layer features are depicted in figure 6 for an accelerator configuration. For simplicity, the thermal and momentum boundary layers are both assumed to have a thickness, $\delta$. In addition, current constriction is indicated at the electrode surface where attachment occurs through short

arcs spanning the anode thickness, $\delta_{a}$, and the cathode thickness, $\delta_{c}$. Note that the illustration shows a concentration of current due to the Hall effect at the upstream end of the cathode and the downstream end of the anode in accordance with experimental observations for accelerators. ${ }^{19,20}$

The effective electrode voltage drop, $V_{d}$, is defined as the potential difference by which the voltage between opposite electrodes is increased (due to boundary layer effects) with respect to the fully homogeneous case. The increase in $V_{d}$ is quantified through an integration of the transverse electric field defect across the electrode boundary layers. For convenience, $V_{d}$ is split into separate components,

$$
V_{d}=V_{a}+V_{c}+V_{g},
$$

where the anode voltage fall, $V_{a}$; the cathode voltage fall, $V_{c}$; and the gasdynamic voltage drop, $V_{g}$, are defined by

$$
\begin{aligned}
V_{a} & =\int_{0}^{\delta_{a}}\left(E_{y}-E_{y 0}\right) d y \\
V_{c} & =\int_{h-\delta_{c}}^{h}\left(E_{y}-E_{y 0}\right) d y \\
V_{g} & =\int_{\delta_{a}}^{\delta}\left(E_{y}-E_{y 0}\right) d y+\int_{h-\delta}^{h-\delta_{c}}\left(E_{y}-E_{y 0}\right) d y .
\end{aligned}
$$

Note that the zero subscript refers to the homogeneous core flow conditions.

Factors contributing to the anode and cathode voltage falls include the arc attachment region and the potential associated with electron emission from cold electrode material. In general, the voltage drop in the arc columns is negligible since the arc length is small compared to the boundary layer thickness, and the anode electrode fall is negligible since it does not have to emit electrons. Thus, $V_{a} \approx 0$. The cathode does emit electrons, however, and the voltage drop associated with this process can only be approximated in an empirical fashion. For most materials, $V_{c}$ is approximately 10 to $20 \mathrm{~V}$. 
The gasdynamic voltage drop may be evaluated by applying the generalized Ohm's law in the boundary layer to eliminate $E_{y}$. In this particular case, the appropriate form for $E_{y}$ may be obtained from equation (9) by neglecting $\Delta$ :

$$
E_{y}=\left(\frac{1+\Omega^{2}}{\Sigma}\right) j_{y}-\Omega E_{x}+u B
$$

Assuming $\Omega$ is invariant, $V_{g}$ takes the form

$$
\begin{aligned}
V_{g}= & \int_{\delta_{a}}^{\delta}\left\{\left[\frac{j_{y}}{\Sigma / 1+\Omega_{0}^{2}}+u B\right]-\left[\frac{j_{y}}{\Sigma_{0} / 1+\Omega_{0}^{2}}+u_{0} B\right]\right\} d y \\
& +\int_{h-\delta}^{h-\delta_{c}}\left\{\left[\frac{j_{y}}{\Sigma / 1+\Omega_{0}^{2}}+u B\right]-\left[\frac{j_{y}}{\Sigma_{0} / 1+\Omega_{0}^{2}}+u_{0} B\right]\right\} d y .
\end{aligned}
$$

And, it is convenient to split $V_{g}$ into two parts

$$
V_{\Sigma}=\frac{2 j_{y}}{\Sigma_{0}^{\prime}} \int_{\delta_{a}}^{\delta}\left[\frac{\Sigma_{0}^{\prime}}{\Sigma^{\prime}}-1\right] d y
$$

and

$$
V_{u}=2 u_{0} B \int_{\delta_{c}}^{\delta}\left[\frac{u}{u_{0}}-1\right] d y
$$

where $V_{\Sigma}$ and $V_{u}$ account separately for the decrease of $\Sigma$ and induced voltage in the boundary layer, and $\Sigma^{\prime}=\Sigma /\left(1+\Omega_{0}^{2}\right)$. These integrals may be further simplified by defining $\xi=y / \delta$ and assuming $\delta_{a}=\delta_{c}$ to yield

$$
V_{\Sigma}=\frac{2 \delta j_{y}}{\Sigma_{0}^{\prime}} \int_{\xi_{c}}^{1}\left[\frac{\Sigma_{0}^{\prime}}{\Sigma^{\prime}}-1\right] d \xi
$$

and

$$
V_{u}=2 \delta u_{0} B \int_{\xi_{c}}^{1}\left[\frac{u}{u_{0}}-1\right] d \xi
$$

The lower limit of integration $\xi_{c}=\delta_{C} / \delta$ is the distance from the electrode surface where the current becomes constricted. 
Evaluation of these integrals is facilitated by the introduction of model boundary layer profiles for velocity and temperature. ${ }^{21}$ For example, the velocity profile in a turbulent boundary layer is well approximated by the power law $u / u_{0}=(y / \delta)^{1 / n}$, where $n \approx 7$. If this relation is substituted into the integral for $V_{u}$ and $\xi_{c}=0$ is used as the lower limit of integration (since $u$ goes to zero at the wall), we obtain

$$
V_{u} \approx 2 u_{0} B \delta /(1+n)
$$

Evaluation of the integral for $V_{\Sigma}$ is complicated by the fact that $\Sigma_{0}^{\prime} / \Sigma^{\prime}$ increases steeply near the cold electrode surface, which makes the integral extremely sensitive to the lower limit of integration. It is assumed that electrical breakdown leading to arc attachment occurs when $j_{\mathrm{y}} / \Sigma^{\prime}$ exceeds the critical electric field strength, $E_{c}$. In this case, the limit, $\xi_{c}$, corresponds to a critical conductivity ratio, $\omega_{c}$, where

$$
\omega_{c}=\frac{\Sigma_{c}^{\prime}}{\Sigma_{0}^{\prime}}=\frac{j_{y} / E_{c}}{\Sigma_{0}^{\prime}}
$$

Experience shows that very near the wall the thermal boundary layer may be approximated by the power law $T / T_{0}=(y / \delta)^{1 / m}$, where $m=18-25$. Furthermore, it has also been established that the electrical conductivity can be approximated by the simple power law $\Sigma^{\prime} / \Sigma_{0}^{\prime}=\left(T / T_{0}\right)^{a}\left(p / p_{0}\right)^{b}$, where $a=10-20$ and $b=-1 / 2$. Thus, for negligible pressure variations, the integral for $V_{\Sigma}$ can be expressed in the simplified form

$$
V_{\Sigma}=\frac{2 \delta j_{y}}{\Sigma_{0}^{\prime}} \int_{\xi_{c}}^{1}\left[\xi^{-a / m}-1\right] d \xi
$$

The calculation procedure is as follows. For a given value of $j_{y} / \Sigma_{0}^{\prime}$ and a specified $E_{c}$, it is possible to compute $\omega_{c}$, which may be used in turn to compute $T_{c} / T_{0}=\omega_{c}^{1 / a}$. Then, the lower limit of integration may be determined from the relation $\xi_{c}=\left(T_{c} / T_{0}\right)^{m}$ and the integral of equation (65) may be evaluated. For illustrative purposes, calculated values for $\xi_{c}$ and $V_{\Sigma} / 2 \delta$ are shown in figure 7 as a function of $j_{y} / \Sigma^{\prime}{ }_{0}$, assuming $E_{c}=40 \mathrm{kV} / \mathrm{cm}, n=7, m=18$, and $a=10$. Note that $V_{\Sigma} \propto j_{y}$ in accordance with empirical observations. 


\section{PRACTICAL DESIGN CONSTRAINTS}

In a real device, practical design constraints limit the available operating range. The axial electric field, for instance, must be limited due to breakdown considerations. If the axial electric field becomes too large, inter-electrode arcing can occur, and the resulting shorts can degrade device performance. Furthermore, high heat dissipation in these concentrated arc discharges can cause severe erosion of wall material and dramatic reduction in channel operating life.

Experience has shown that a realistic upper limit for the axial electric field is no more than $100 \mathrm{~V} / \mathrm{cm}$. The existence of such a design constraint is illustrated by the dashed lines marked $\pm \bar{E}_{x, l i m}$ on the performance map of figure 5. This implies that DCW accelerators operating with large $\Omega$ are confined to low power-density operation. High power densities are attainable with low $\Omega$ at the expense of decreased efficiency.

An additional design constraint arises from the observation that excessive heat generation and material erosion will occur if the current density entering the electrode surface becomes too large. The actual limiting value depends on electrode material and geometry, but experience indicates good channel durability for values up to about $10 \mathrm{~A} / \mathrm{cm}^{2}$. For high power-density applications having short lifetime requirements, this value can be exceeded considerably. 


\section{CONCLUSIONS}

The classical cross-plane averaged performance theory for DCW MHD accelerators has been extended to include a current-dependent sheath layer voltage drop. This approach yields analytical performance relationships and diagrams that can be used to illuminate the rudimentary behavior of these devices and underscore the fundamental interplay of basic parameters. This simplified theoretical treatment is not intended for detailed performance prediction of practical devices but rather to aid in developing an intuitive understanding of device operation of general value to analysts, designers, and experimentalists, alike. Despite its limitations, the theory can be extremely useful in defining anticipated performance ranges while accounting for critical nonideal effects. 


\section{REFERENCES}

1. Litchford, R.J.; Cole, J.W.; Lineberry, J.T.; Chapman, J.N.; Schmidt, H.J.; and Lineberry, C.W.: "Magnetohydrodynamic Augmented Propulsion Experiment: I. Performance Analysis and Design," AIAA Paper No. 2002-2184, 33rd AIAA Plasmadynamics and Lasers Conference, Maui, HI. May 20-23, 2002.

2. Powers, W.L.; Dicks, J.B.; and Snyder, W.T.: "A Graphical Presentation of MHD Accelerator and Generator Performance Characteristics," AIAA J., Vol. 5, No. 12, pp. 2232-2236, December 1967.

3. Wu, Y.C.L.: "Performance Theory of Diagonal Conducting Wall MHD Generators," AIAA J., Vol. 14, No. 10, pp. 1362-1368, October 1976.

4. Buende, R.; Muntenbruch, H.; Raeder, J.; Volk, R.; and Zauk1, G.: MHD Power Generation: Selected Problems of Combustion MHD Generators, J. Raeder (ed.), Springer-Verlag, Berlin, Germany, 1975.

5. Bityurin, V.A.; Zatelepin, V.N.; and Lyubimov, G.A.: "A Consideration of Some Three-Dimensional Effects in MHD Channels," Proceedings of 16th Symposium on Engineering Aspects of Magnetohydrodynamics (SEAM), Pittsburgh, PA, May 1977.

6. Ahluwalia, R.K.; Vanka, S.P.; Im, K.H.; and Zwick, S.A.: "Formulation and Assessment of a CrossPlane Electrical Model for Magnetohydrodynamic Channels," J. Energy, Vol. 6, No. 5, pp. 314-322, October 1982.

7. De Montardy, A.: "MHD Generator with Series-Connected Electrodes," Proceedings of the International Symposium on MHD Electrical Power Generation, Newcastle upon Tyne, England, Paper No. 19, September 1962.

8. Dicks, J.B.: "Design and Operation of Open Cycle Hall Current Neutralized MHD Accelerators and Generators with Diagonal Conducting Strip Walls," Proceedings of 5th Symposium on Engineering Aspects of Magnetohydrodynamics (SEAM), Massachusetts Institute of Technology, Cambridge, MA, April 1964.

9. Dicks, J.B.: "Improvements in Design of MHD Accelerator Channels for Aerodynamic Purposes," in Arc Heaters and MHD Accelerators for Aerodynamic Purposes, AGARDograph 84: Supplemental Volume, Proceedings of AGARD Specialists Meeting, North Atlantic Treaty Organization, Advisory Group for Aeronautical Research and Development, pp. 127-174, September 1964.

10. Schlueter, A.: “Dynamik des Plasmas I,” Z. Naturforschung, Vol. 5a, p. 72, 1950; Vol. 6a, pp. 73-78, 1951. 
11. Cowling, T.G.: Chapter 6 in Magnetohydrodynamics, Interscience Publishers, Inc., New York, NY, p. 107, 1957.

12. Sherman, A.: "The Generalized Ohm's Law for a Partially Ionized Gas," Internal Report DF59FPD807, General Electric Company, October 1959.

13. Polovin, R.V.; and Cherkasova, K.P.: "Magnetohydrodynamic Description of a Plasma," Soviet Physics—Technical Physics, Vol. 7, No. 6, pp. 475-479, December 1962.

14. Liubimov, G.A.: "On the Form of Ohm's Law in Magnetohydrodynamics," Soviet Mathematics: Applied Mathematics and Mechanics, Vol. 25, No. 4, pp. 913-929, February 1962.

15. Rosa, R.J.: "Hall and Ion Slip Effects in a Nonuniform Gas," Phys. Fluids, Vol. 5, No. 9, pp. 10811090, September 1962.

16. Wu, Y.C.L.; Dicks, J.B.; Crawford, L.W.; Muchlhauser, J.W.; Scott, M.A.; and Snood, N.: "Theoretical and Experimental Studies of MHD Power Generation With Char," Proceedings of 12th Symposium on Engineering Aspects of Magnetohydrodynamics (SEAM), Argonne National Laboratory, Argonne, IL, pp. II.1.1-II.1.14, March 1972.

17. Wu, Y.C.L.; Rajogopal, G.; and Dicks, J.B.: "Investigation of Open-Cycle MHD Power Generation," AIAA Paper No. 74-175, 12th American Institute of Aeronautics and Astronautics Aerospace Sciences Meeting, Washington, D.C., January 1974.

18. Shanklin, R.V., III; and Nimmo, R.A.: "Report on the Status and Results of the KIVA-I Open Cycle MHD Generator System," Proceedings of 14th Symposium on Engineering Aspects of Magnetohydrodynamics (SEAM), University of Tennessee Space Institute, Tullahoma, TN, pp. I.8.1-I.8.5, April 1974.

19. Demetriades, S.T.; and Lenn, P.D.: "Electrical Discharge Across a Supersonic Jet of Plasma in Transverse Magnetic Field," AIAA J., Vol. 1, pp. 234-236, 1963.

20. Denison, M.R.; and Zeimer, R.W.: "Investigation of the Phenomena in Cross-Field Plasma Accelerators," in Physico-Chemical Diagnostics of Plasmas, Proceedings of the Fifth Biennial Gas Dynamics Symposium, T.P. Anderson, R.W. Springer, and R.C. Warder (eds.), Northwestern University Press, Evanston, IL, pp. 201-232, 1964.

21. Schlichting, H.: Boundary Layer Theory, Sixth Edition, McGraw-Hill, New York, NY, 1968. 
Table 1. Summary of electrical parameter relationships for diagonalized cross-field MHD devices.*

\begin{tabular}{|c|c|c|}
\hline Electrical Parameter & In terms of applied electric field, $E_{x}$ & In terms of applied electric current, $I$ \\
\hline \multirow{2}{*}{$j_{x}$} & $\underline{(1-\Omega \varphi) \Sigma E_{x}+\Omega \Sigma u B(1+\Delta)}$ & $\underline{(1-\Omega \varphi) I / A+\varphi \Sigma u B(1+\Delta)}$ \\
\hline & $1+\Omega^{2}$ & $1+\varphi^{2}$ \\
\hline \multirow{2}{*}{$j_{y}$} & $(\Omega+\varphi) \Sigma E_{x}-\Sigma u B(1+\Delta)$ & $\underline{(\Omega+\varphi) I / A-\Sigma u B(1+\Delta)}$ \\
\hline & $1+\Omega^{2}$ & $1+\varphi^{2}$ \\
\hline \multirow{2}{*}{$E_{x}$} & \multirow{2}{*}{$E_{x}$} & $\left(1+\Omega^{2}\right) I / A+(\varphi-\Omega) \Sigma u B(1+\Delta)$ \\
\hline & & $\Sigma\left(1+\varphi^{2}\right)$ \\
\hline$E_{y}$ & $\varphi E_{x}$ & $\varphi E_{x}$ \\
\hline$I$ & $\frac{\left(1+\varphi^{2}\right) \Sigma E_{x} A+(\Omega-\varphi) \Sigma u B(1+\Delta) A}{1+\Omega^{2}}$ & $I$ \\
\hline
\end{tabular}

*The sign of $\varphi$ and $\Delta$ depends on the mode of operation. Generator mode: $\varphi<0$ and $-1 \leq \Delta \leq 0$; accelerator mode: $\varphi>0$ and $\Delta \geq 0$.

Table 2. Summary of mean power relationships for diagonalized cross-field MHD devices.*

\begin{tabular}{ccc}
\hline \hline Power Parameter & In terms of applied electric field, $E_{x}$ & In terms of applied electric current, $I$ \\
\hline$P=\mathbf{j} \cdot \mathbf{E}$ & $\Sigma E_{x}^{2}\left[\frac{\left(1+\varphi^{2}\right)+(\Omega-\varphi) u B(1+\Delta) / E_{x}}{1+\Omega^{2}}\right]$ & $\frac{I^{2}}{\Sigma A^{2}}\left[\frac{\left(1+\Omega^{2}\right)+(\varphi-\Omega) \Sigma u B(1+\Delta) A / I}{1+\varphi^{2}}\right]$ \\
$P_{p}=\mathbf{u} \cdot(\mathbf{j} \times \mathbf{B})_{x}$ & $\Sigma E_{x} u B\left[\frac{(\Omega+\varphi)-u B(1+\Delta) / E_{x}}{1+\Omega^{2}}\right]$ & $\frac{u B I}{A}\left[\frac{(\Omega+\varphi)-\Sigma u B(1+\Delta) A / I}{1+\varphi^{2}}\right]$ \\
$\eta_{a}=\frac{\mathbf{u} \cdot(\mathbf{j} \times \mathbf{B})_{x}}{\mathbf{j} \cdot \mathbf{E}}=\frac{1}{\eta_{g}}$ & $\frac{u B}{E_{x}}\left[\frac{(\Omega+\varphi)-u B(1+\Delta) / E_{x}}{\left(1+\varphi^{2}\right)+(\Omega-\varphi) u B(1+\Delta) / E_{x}}\right]$ & $\frac{\Sigma u B}{I / A}\left[\frac{(\Omega+\varphi)-\Sigma u B(1+\Delta) A / I}{\left(1+\Omega^{2}\right)+(\varphi-\Omega) \Sigma u B(1+\Delta) A / I}\right]$ \\
\hline \hline
\end{tabular}

*The sign of $\varphi$ and $\Delta$ depends on the mode of operation. Generator mode: $\varphi<0$ and $-1 \leq \Delta \leq 0$; accelerator mode: $\varphi>0$ and $\Delta \geq 0$.

Table 3. Reference conditions for variable $\Delta$.

\begin{tabular}{lcccc}
\hline \hline Mode & $\Delta^{\prime}$ & $\Delta^{\prime \prime}$ & $I^{\prime}$ & $I^{\prime \prime}$ \\
\hline Generator & $\Delta_{o}$ & $\Delta_{s}$ & $I_{o}=0$ & $I_{s}$ \\
Accelerator & $\Delta_{z}=\Delta_{s}$ & $\Delta_{n}=0$ & $I_{z}$ & $I_{n}$ \\
\hline \hline
\end{tabular}




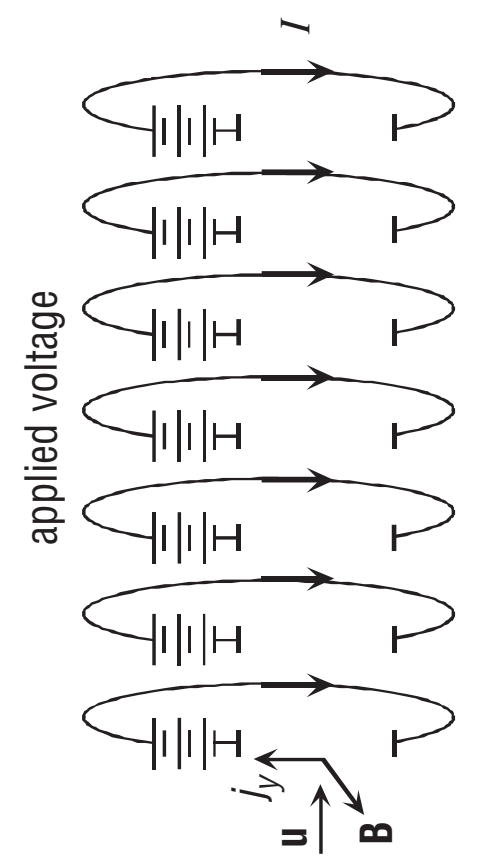

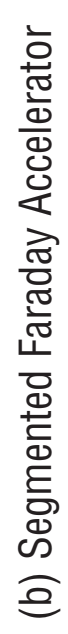
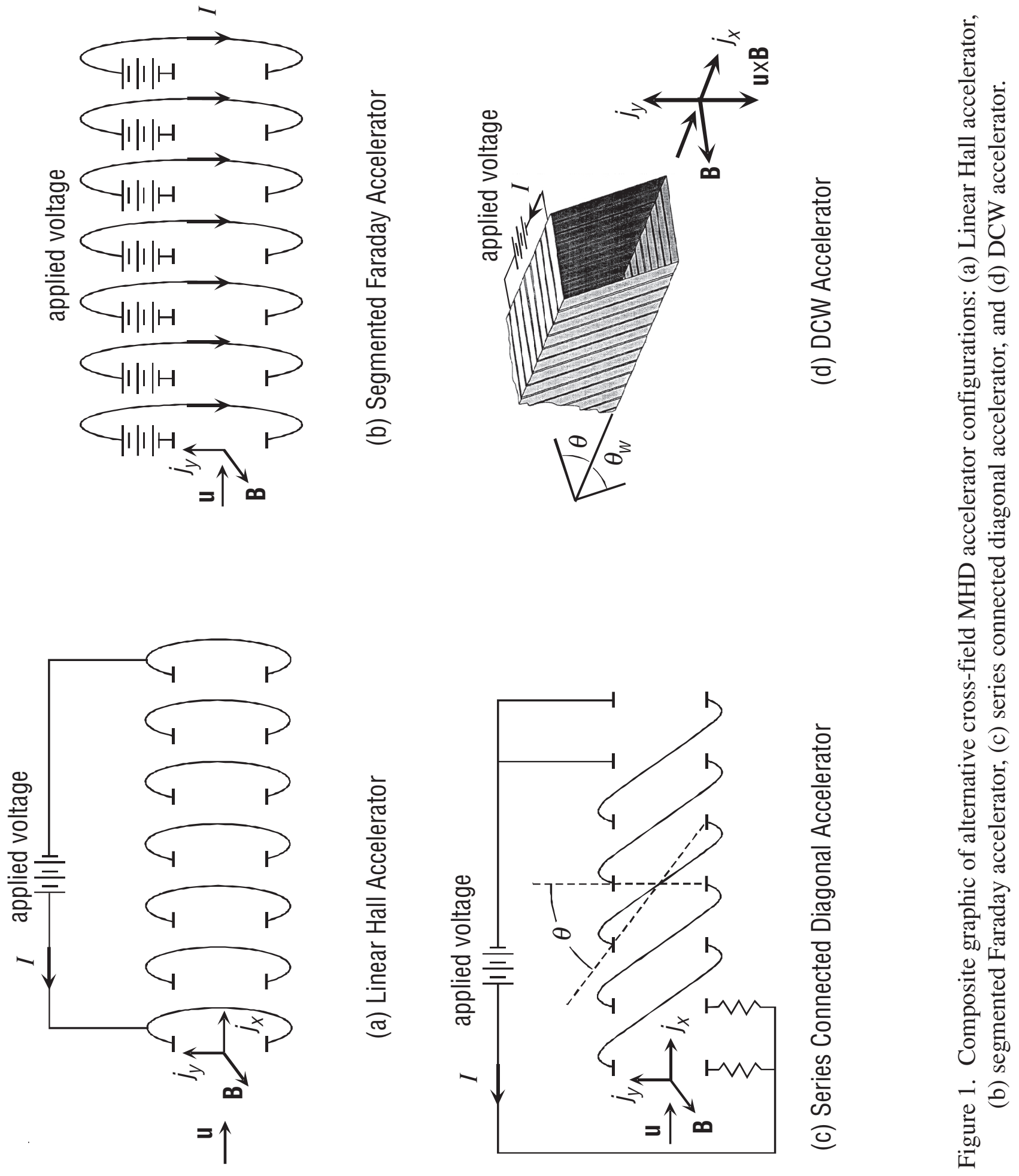

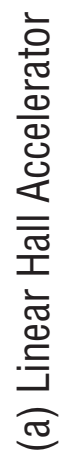

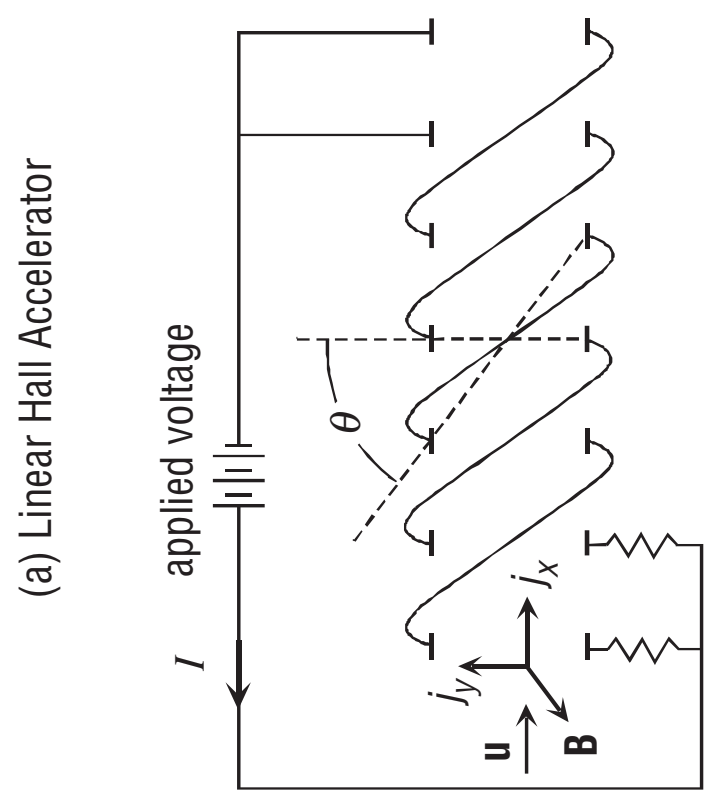

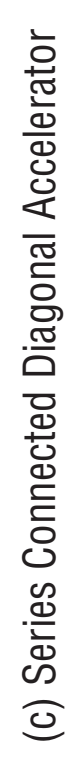

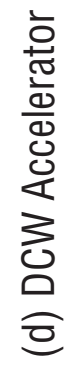




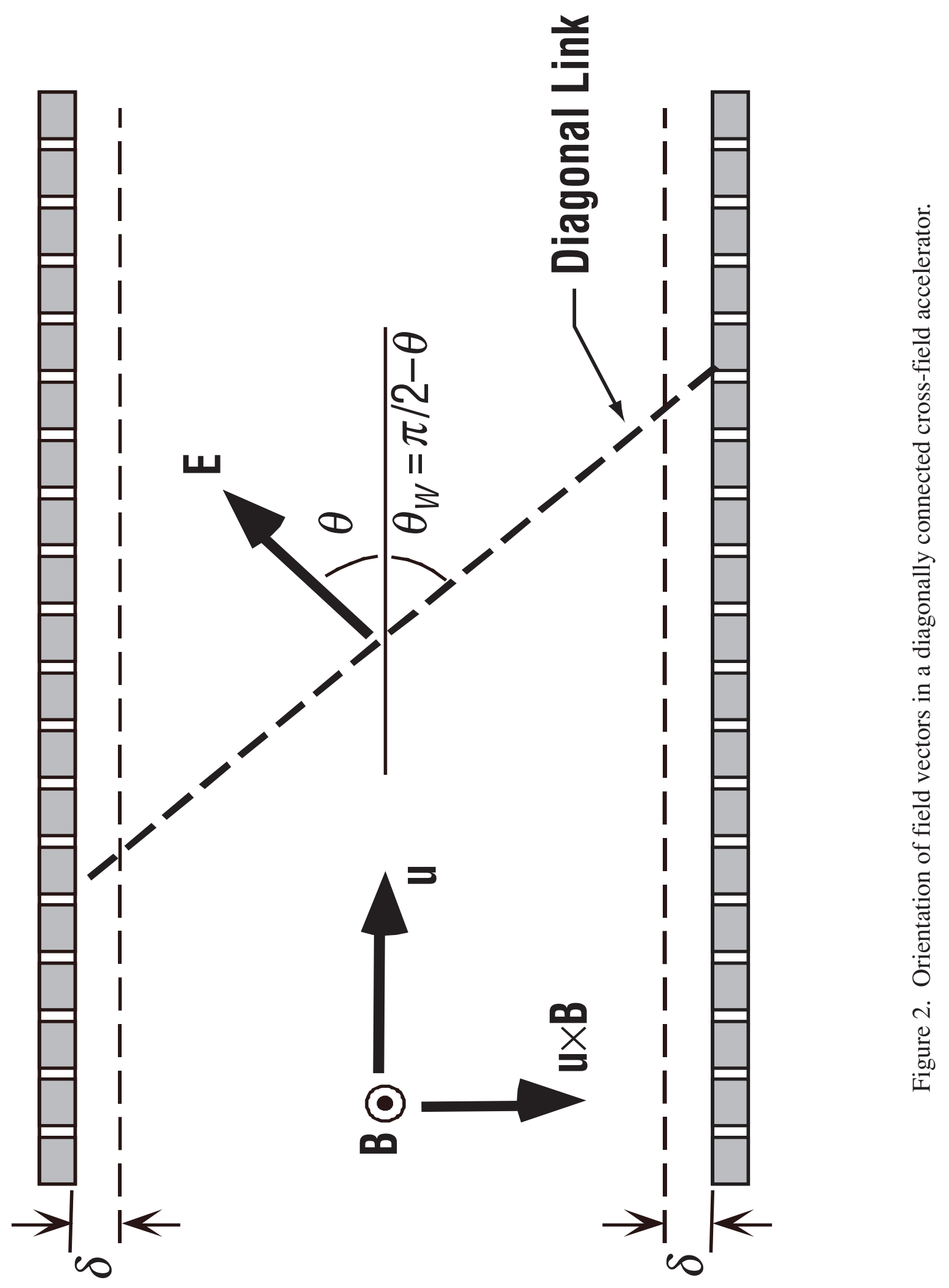



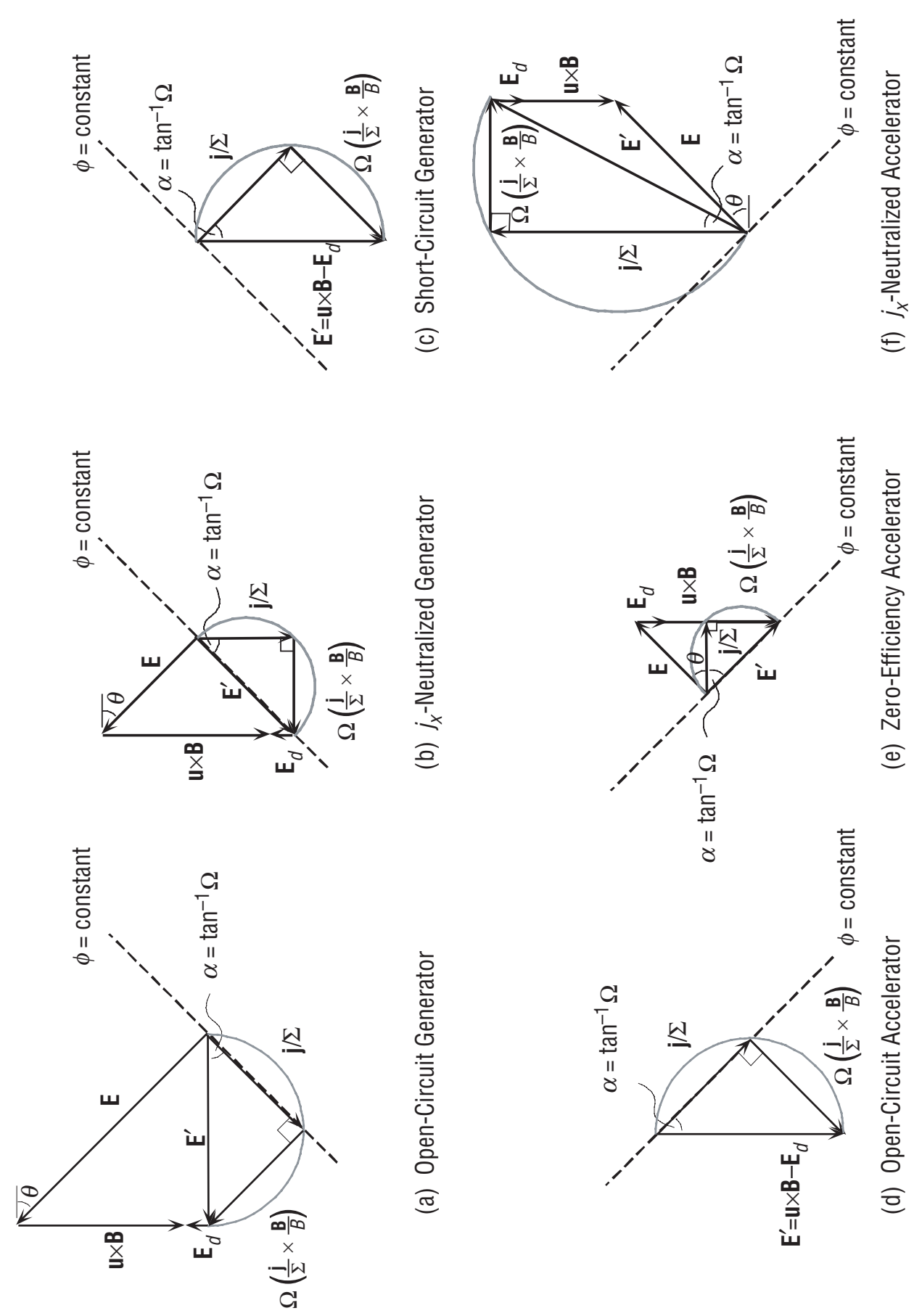

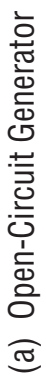

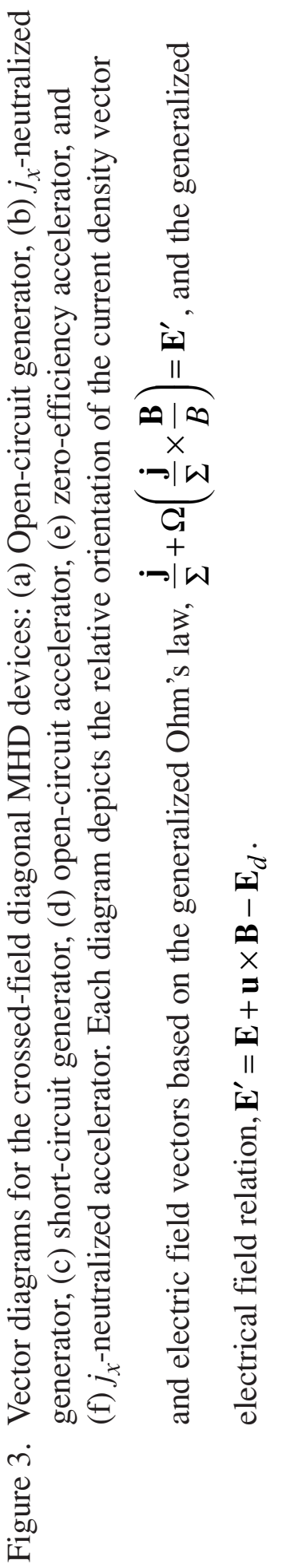




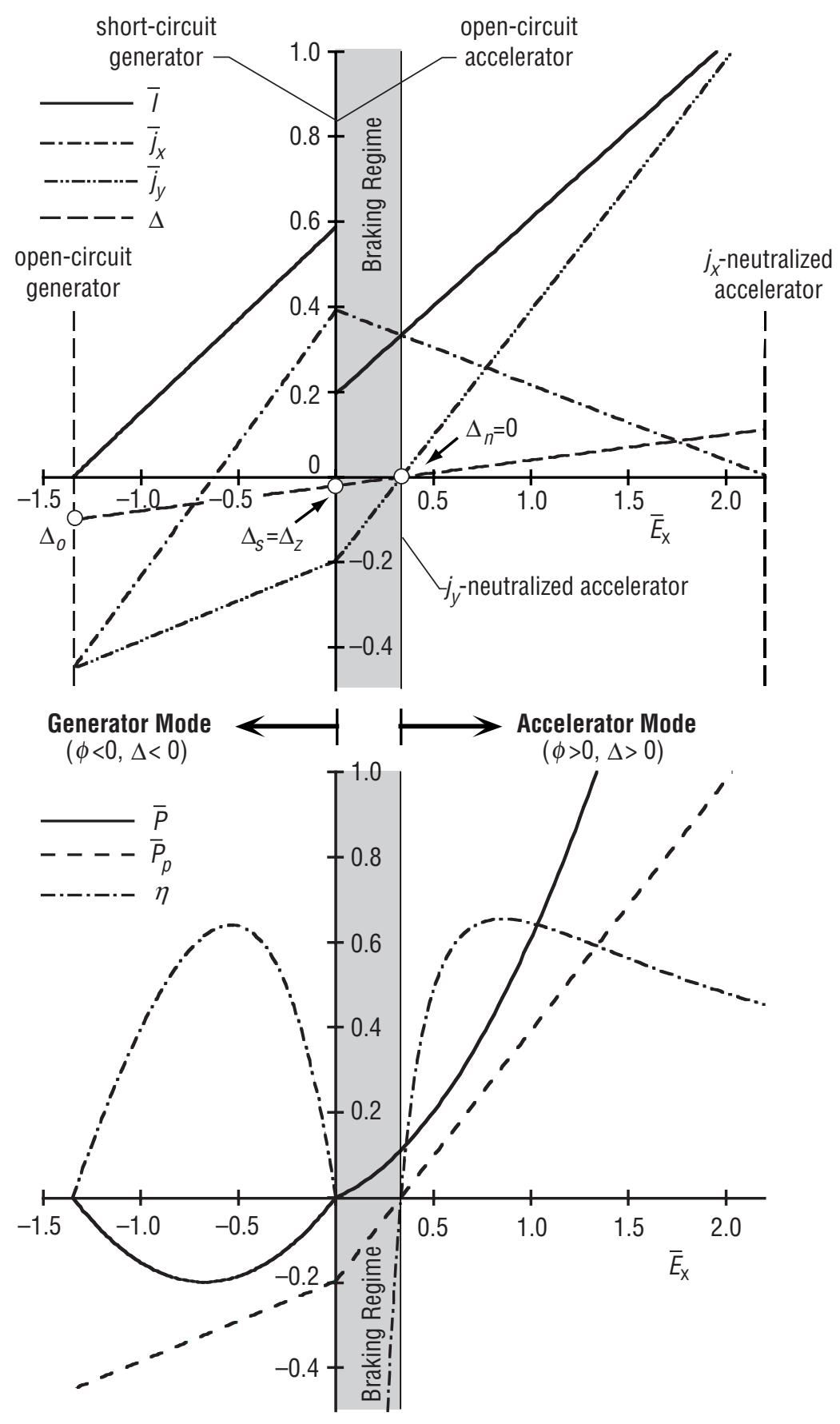

Figure 4. Variation of performance parameters based on representative values for flow/field conditions, material functions, and wall angle. Assumes that $\varphi= \pm 1, B=4 \mathrm{~T}, \Sigma=100, \Omega=2, u=3 \mathrm{~km} / \mathrm{s}, \Delta_{o}=-0.1$, and $\Delta_{S}=-0.02$. 


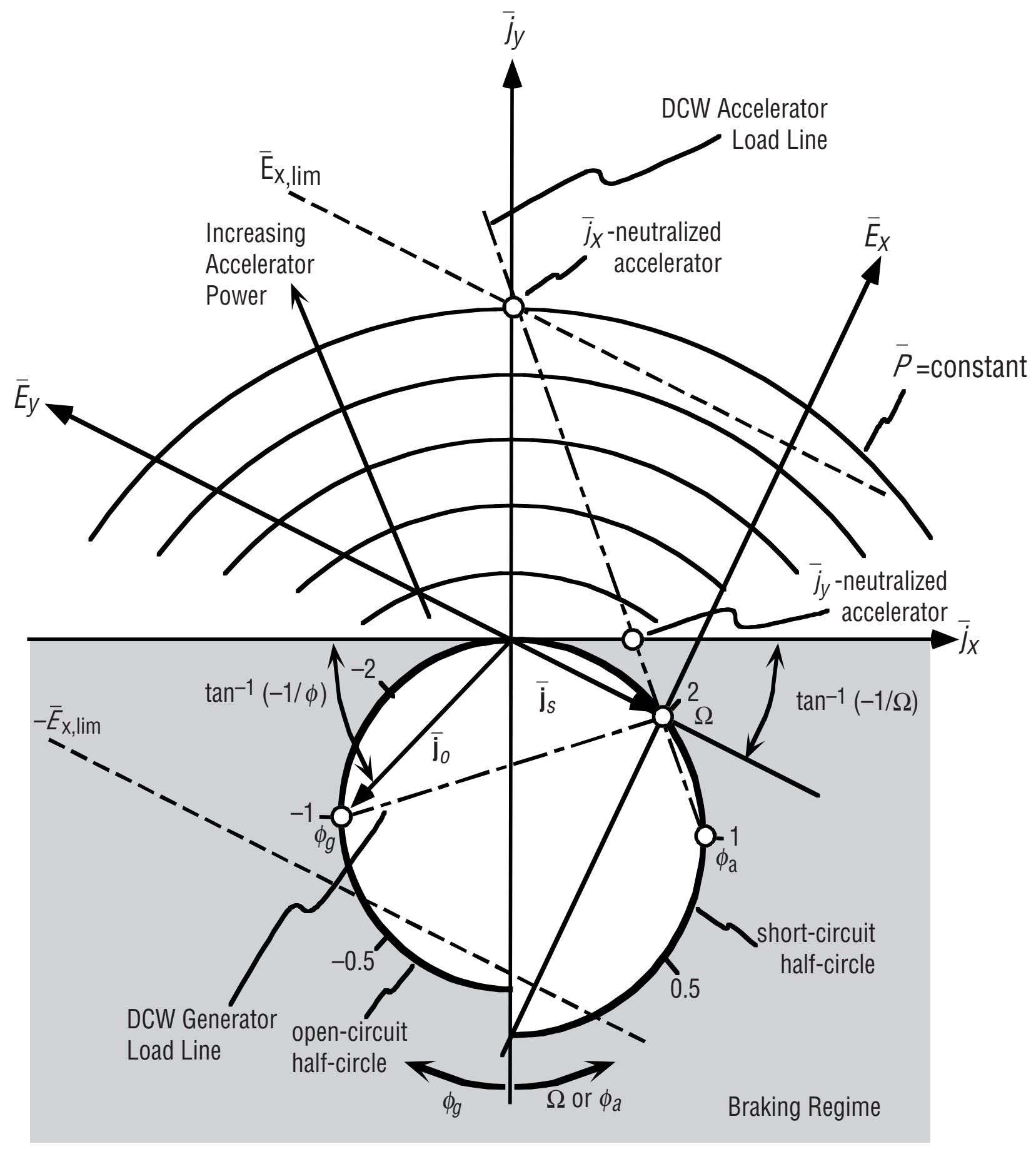

Figure 5. Performance map for DCW MHD devices with current-dependent voltage drop effect. 


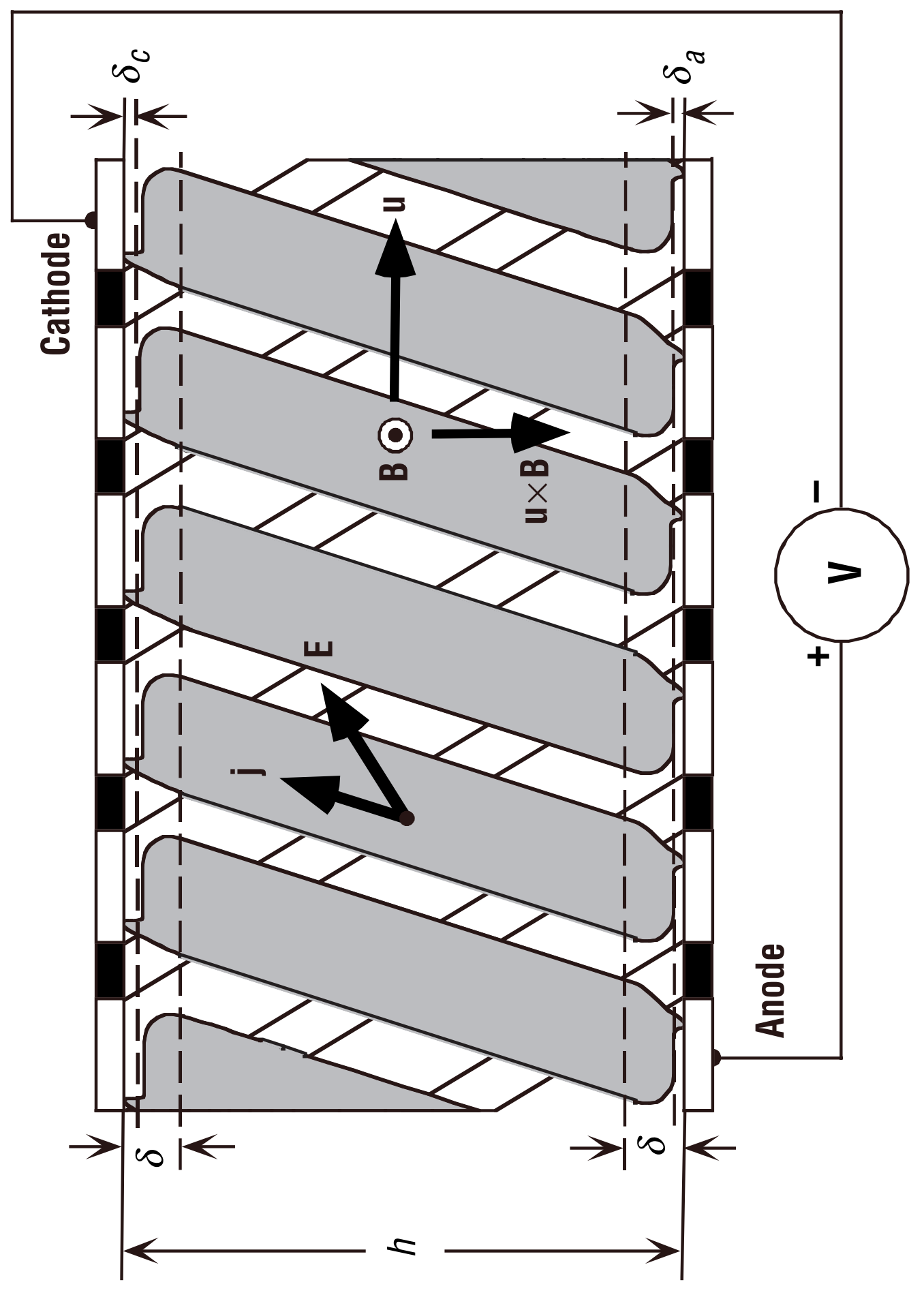

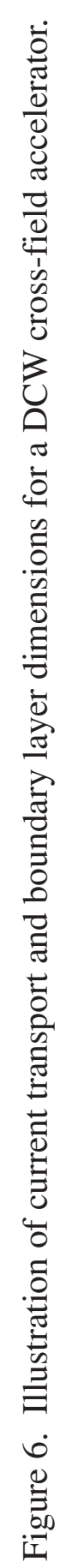



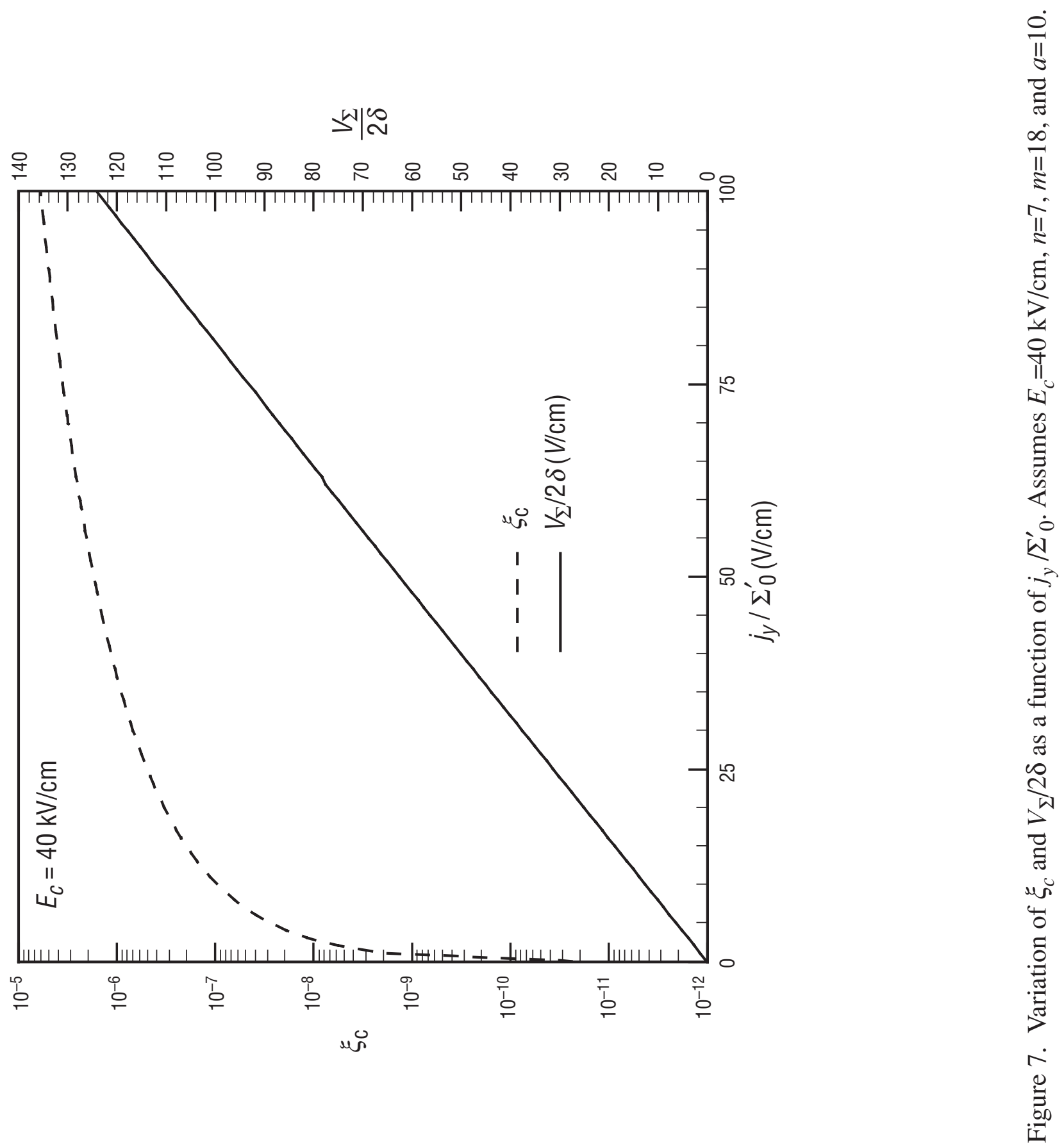


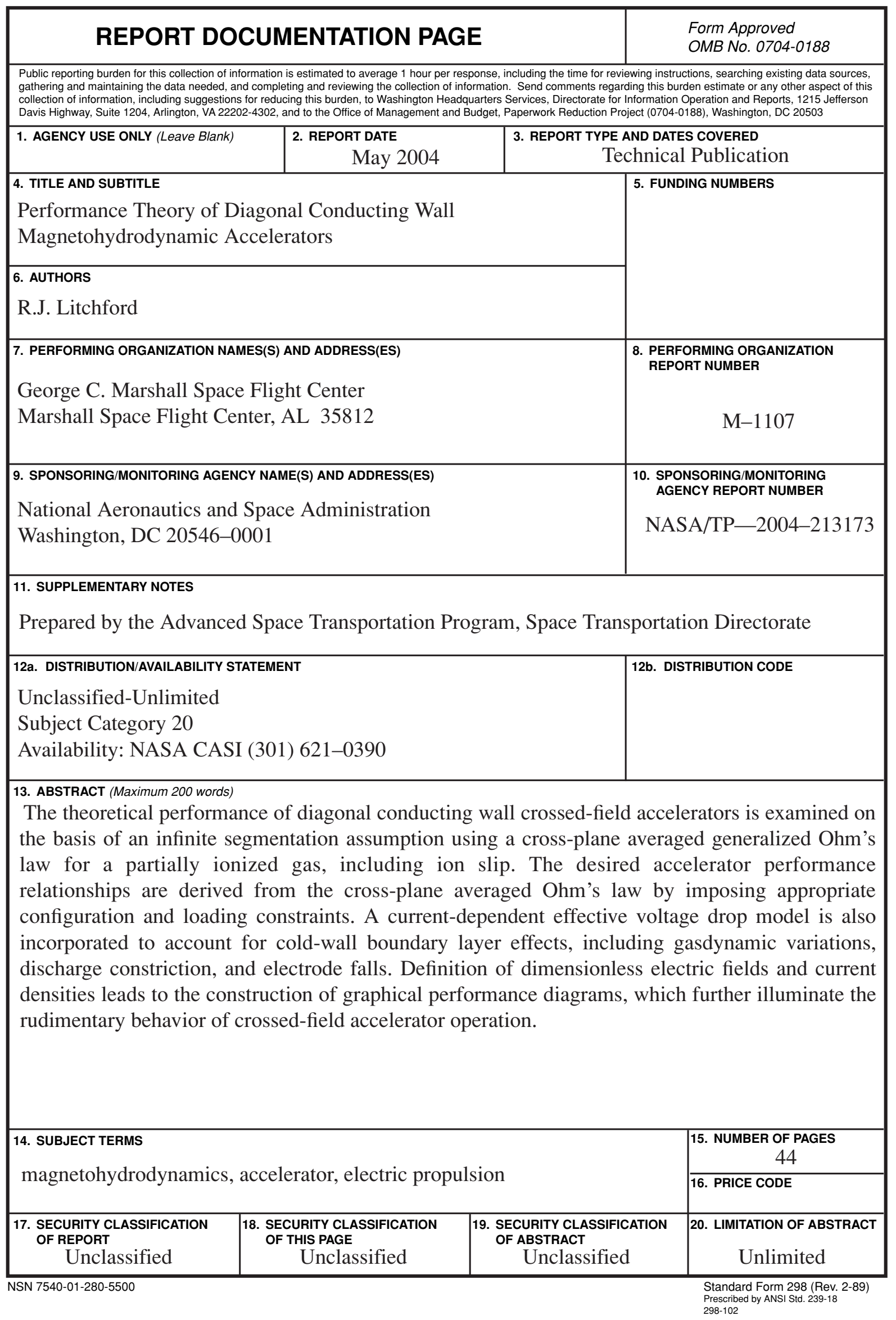

